\title{
Noncommutative Generalizations of Theorems of Cohen and Kaplansky
}

\author{
Manuel L. Reyes
}

Received: 12 July 2010 / Accepted: 3 January 2011 / Published online: 9 February 2011

(C) The Author(s) 2011. This article is published with open access at Springerlink.com

\begin{abstract}
This paper investigates situations where a property of a ring can be tested on a set of "prime right ideals." Generalizing theorems of Cohen and Kaplansky, we show that every right ideal of a ring is finitely generated (resp. principal) iff every "prime right ideal" is finitely generated (resp. principal), where the phrase "prime right ideal" can be interpreted in one of many different ways. We also use our methods to show that other properties can be tested on special sets of right ideals, such as the right artinian property and various homological properties. Applying these methods, we prove the following noncommutative generalization of a result of Kaplansky: a (left and right) noetherian ring is a principal right ideal ring iff all of its maximal right ideals are principal. A counterexample shows that the left noetherian hypothesis cannot be dropped. Finally, we compare our results to earlier generalizations of Cohen's and Kaplansky's theorems in the literature.
\end{abstract}

Keywords Point annihilators • Cocritical right ideals • Cohen's theorem • Right noetherian rings $\cdot$ Kaplansky's theorem $\cdot$ Principal right ideals

Mathematics Subject Classifications (2010) Primary 16D25 • 16P40 • 16P60; Secondary $16 \mathrm{~N} 60$

Presented by Kenneth R. Goodearl.

The author was supported in part by a Ford Foundation Predoctoral Diversity Fellowship. This work is a portion of his Ph.D. thesis at the University of California, Berkeley.

M. L. Reyes

Department of Mathematics, University of California, Berkeley, CA 94720-3840, USA

Present Address:

M. L. Reyes $(\varangle)$

Department of Mathematics, University of California, San Diego,

9500 Gilman Drive, \#0112, La Jolla, CA 92093-0112, USA

e-mail: m1reyes@math.ucsd.edu 


\section{Introduction}

Two famous theorems from commutative algebra due to I. S. Cohen and I. Kaplansky state that, to check whether every ideal in a commutative ring is finitely generated (respectively, principal), it suffices to test only the prime ideals. Cohen's Theorem appeared as Theorem 2 of [6].

Theorem 1.1 (Cohen's Theorem) A commutative ring $R$ is noetherian iff every prime ideal of $R$ is finitely generated.

Also, we recall a characterization of commutative principal ideal rings due to I. Kaplansky, which appeared as Theorem 12.3 of [18]. Throughout this paper, a ring in which all right ideals are principal will be called a principal right ideal ring, or PRIR. Similarly, we have principal left ideal rings (PLIRs), and a ring which is both a PRIR and a PLIR is called a principal ideal ring, or PIR.

Theorem 1.2 (Kaplansky's Theorem) A commutative noetherian ring $R$ is a principal ideal ring iff every maximal ideal of $R$ is principal.

Combining this result with Cohen's Theorem, Kaplansky deduced the following in Footnote 8 on p. 486 of [18].

Theorem 1.3 (Kaplansky-Cohen Theorem) A commutative ring $R$ is a principal ideal ring iff every prime ideal of $R$ is principal.

(We refer to this result as the Kaplansky-Cohen Theorem for two reasons. The primary and most obvious reason is that it follows from a combination of the above results due to Cohen and Kaplansky. But we also use this term because it is a result in the spirit of Cohen's Theorem, that was first deduced by Kaplansky.)

The unifying theme of this paper is the generalization of the above theorems to noncommutative rings, using certain families of right ideals as our tools. Let us mention a typical method of proof of these theorems, as this will guide our investigation into their noncommutative generalizations. One first assumes that the prime ideals of a commutative ring $R$ are all finitely generated (or principal), but that there exists an ideal of $R$ that is not f.g. (or principal). One then passes to a "maximal counterexample" by Zorn's Lemma and proves that such a maximal counterexample is prime. This contradicts the assumption that all primes have the relevant property, proving the theorem.

These "maximal implies prime" theorems were systematically studied in [29] from the viewpoint of certain families of ideals, called Oka families. In particular, the Cohen and Kaplansky-Cohen theorems were recovered in [29, p. 3017]. These families were generalized to noncommutative rings in [33], where we defined $O \mathrm{ka}$ families of right ideals. This resulted in a noncommutative generalization of Cohen's Theorem in [33, Theorem 6.2], stating that a ring $R$ is right noetherian iff its completely prime right ideals are all finitely generated. In the present paper, we will improve upon this result, providing smaller "test sets" of right ideals that can be checked to determine if a ring is right noetherian. In addition, we will provide generalizations of Kaplansky's Theorem 1.2 and the Kaplansky-Cohen Theorem 1.3. 
We begin by reviewing the relevant results from [29] in Section 2. This includes an introduction to the notions of right Oka families, classes of cyclic modules closed under extensions, completely prime right ideals, the Completely Prime Ideal Principle (CPIP), and the CPIP Supplement. Our work in Sections 3 and 4 addresses the following question: what are some sufficient conditions for all right ideals of a ring to lie in a given right Oka family? In Section 3 we develop the idea of a (noetherian) point annihilator set in order to deal with this problem. Then in Section 4 we prove the Point Annihilator Set Theorem 4.1. Along with its consequences, such as Theorem 4.3, this theorem gives sufficient conditions for a property of right ideals to be testable on a smaller set of right ideals. We achieve a generalization of Cohen's Theorem in Theorem 4.5. This result is "flexible" in the sense that, in order to check whether a ring is right noetherian, one can use various test sets of right ideals (in fact, certain point annihilator sets will work). One important such set is the cocritical right ideals. Other consequences of the Point Annihilator Set Theorem are also investigated.

Next we consider families of principal right ideals in Section 5. Whereas the family of principal ideals of a commutative ring is always an Oka family, it turns out that the family $\mathcal{F}_{\text {pr }}$ of principal right ideals can fail to be a right Oka family in certain noncommutative rings. By defining a right Oka family $\mathcal{F}_{\mathrm{pr}}^{\circ}$ that "approximates" $\mathcal{F}_{\mathrm{pr}}$, we are able to provide a noncommutative generalization of the Kaplansky-Cohen Theorem in Theorem 5.11. As before, a specific version of this theorem is the following: a ring is a principal right ideal ring iff all of its cocritical right ideals are principal.

In Section 6 we sharpen our versions of the Cohen and Kaplansky-Cohen Theorems by considering families of right ideals that are closed under direct summands. This allows us to reduce the "test sets" of the Point Annihilator Set Theorem 4.3 to sets of essential right ideals. For instance, to check if a ring is right noetherian or a principal right ideal ring, it suffices to test the essential cocritical right ideals. Other applications involving homological properties of right ideals are considered.

We work toward a noncommutative generalization of Kaplansky's Theorem 1.2 in Section 7. The main result here is Theorem 7.9, which states that a (left and right) noetherian ring is a principal right ideal ring iff its maximal right ideals are principal. Notably, our analysis also implies that such a ring has right Krull dimension $\leq 1$. An example shows that the theorem does not hold if the left noetherian hypothesis is omitted.

Finally, we explore the connections between our results and previous generalizations of the Cohen and Kaplansky-Cohen theorems in Section 8. These results include theorems due to V.R. Chandran, K. Koh, G. O. Michler, P. F. Smith, and B. V. Zabavs'kil. Discussing these earlier results affords us an opportunity to survey some previous notions of "prime right ideals" studied in the literature.

\section{Conventions}

All rings are associative and have identity, and all modules and ring homomorphisms are unital. Let $R$ be a ring. We say $R$ is a semisimple ring if $R_{R}$ is a semisimple module. We denote the Jacobson radical of $R$ by $\operatorname{rad}(R)$. We say that $R$ is semilocal (resp. local) if $R / \operatorname{rad}(R)$ is semisimple (resp. a division ring). Given a family $\mathcal{F}$ of 
right ideals in a ring $R$, we let $\mathcal{F}^{\prime}$ denote the complement of $\mathcal{F}$ within the set of all right ideals of $R$. Now fix an $R$-module $M_{R}$. We will write $N \subseteq_{e} M$ to mean that $N$ is an essential submodule of $M$. A proper factor of $M$ is a module of the form $M / N$ for some nonzero submodule $N_{R} \subseteq M$.

\section{Review of Right Oka Families}

In [33], we introduced the following notion of a "one-sided prime."

Definition 2.1 A right ideal $P_{R} \subsetneq R$ is a completely prime right ideal if, for all $a$, $b \in R$,

$$
a P \subseteq P \text { and } a b \in P \Longrightarrow a \in P \text { or } b \in P .
$$

Notice immediately that a two-sided ideal is completely prime as a right ideal iff it is a completely prime ideal (that is, the factor ring is a domain). In particular, the completely prime right ideals of a commutative ring are precisely the prime ideals of that ring.

One way in which these right ideals behave like prime ideals of commutative rings is that right ideals that are maximal in certain senses tend to be completely prime. A more precise statement requires a definition. Given a right ideal $I$ and element $a$ of a ring $R$, we denote

$$
a^{-1} I=\{r \in R: \text { ar } \in I\}
$$

Definition 2.2 A family $\mathcal{F}$ of right ideals in a ring $R$ is an Oka family of right ideals (or a right Oka family) if $R \in F$ and, for any element $a \in R$ and any right ideal $I_{R} \subseteq R$,

$$
I+a R, a^{-1} I \in \mathcal{F} \Longrightarrow I \in \mathcal{F} \text {. }
$$

For a family $\mathcal{F}$ of right ideals in a ring $R$, we let $\mathcal{F}^{\prime}$ denote the complement of $\mathcal{F}$ (the set of right ideals of $R$ that do not lie in $\mathcal{F}$ ), and we let $\operatorname{Max}\left(\mathcal{F}^{\prime}\right)$ denote the set of right ideals of $R$ that are maximal in $\mathcal{F}^{\prime}$. The precise "maximal implies prime" result, which was proved in [33, Theorem 3.6], can now be stated.

Theorem 2.3 (Completely Prime Ideal Principle) Let $\mathcal{F}$ be an Oka family of right ideals in a ring $R$. Then any right ideal $P \in \operatorname{Max}\left(\mathcal{F}^{\prime}\right)$ is completely prime.

A result accompanying the Completely Prime Ideal Principle (CPIP) shows that, for special choices of right Oka families $\mathcal{F}$, in order to test whether $\mathcal{F}$ consists of all right ideals it is enough to check that all completely prime right ideals lie in $\mathcal{F}$. Throughout this paper, a family $\mathcal{F}_{0}$ of right ideals in a ring $R$ is called a semifilter if, whenever $J \in \mathcal{F}$ and $I$ is a right ideal of $R, J \subseteq I$ implies $I \in \mathcal{F}$.

Theorem 2.4 (Completely Prime Ideal Principle Supplement) Let $\mathcal{F}$ be a right Oka family in a ring $R$ such that every nonempty chain of right ideals in $\mathcal{F}^{\prime}$ (with respect to inclusion) has an upper bound in $\mathcal{F}^{\prime}$. Let $\mathcal{S}$ denote the set of completely prime right ideals of $R$. 
- Let $\mathcal{F}_{0}$ be a semifilter of right ideals in $R$. If $\mathcal{S} \cap \mathcal{F}_{0} \subseteq \mathcal{F}$, then $\mathcal{F}_{0} \subseteq \mathcal{F}$.

- For $J_{R} \subseteq R$, if all right ideals in $\mathcal{S}$ containing $J$ (resp. properly containing $J$ ) belong to $\mathcal{F}$, then all right ideals containing $J$ (resp. properly containing $J$ ) belong to $\mathcal{F}$.

- If $\mathcal{S} \subseteq \mathcal{F}$, then $\mathcal{F}$ consists of all right ideals of $R$.

In order to efficiently construct right Oka families, we established the following correspondence in [33, Theorem 4.7]. A class $\mathcal{C}$ of cyclic right $R$-modules is said to be closed under extensions if $0 \in \mathcal{C}$ and, for every short exact sequence $0 \rightarrow L \rightarrow M \rightarrow$ $N \rightarrow 0$ of cyclic right $R$-modules, $L \in \mathcal{C}$ and $N \in \mathcal{C}$ imply $M \in \mathcal{C}$. Given a class of cyclic right $R$-modules, one may construct the following family of right ideals of $R$ :

$$
\mathcal{F}_{\mathcal{C}}:=\left\{I_{R} \subseteq R: R / I \in \mathcal{C}\right\}
$$

Conversely, given a family $\mathcal{F}$ of right ideals in $R$, we construct a class of cyclic $R$-modules

$$
\mathcal{C}_{\mathcal{F}}:=\left\{M_{R}: M \cong R / I \text { for some } I \in \mathcal{F}\right\} \text {. }
$$

Theorem 2.5 Given a class $\mathcal{C}$ of cyclic right $R$-modules that is closed under extensions, the family $\mathcal{F}_{\mathcal{C}}$ is a right Oka family. Conversely, given a right Oka family $\mathcal{F}$, the class $\mathcal{C}_{\mathcal{F}}$ is closed under extensions.

This theorem was used to construct a number of examples of right Oka families in [33]. For us, the most important such example is the finitely generated right ideals: in any ring $R$, the family of finitely generated right ideals of $R$ is a right Oka family (see [33, Proposition 3.7]).

An easy consequence of the above theorem, proved in [33, Corollary 4.9], will be useful throughout this paper.

Corollary 2.6 Let $\mathcal{F}$ be a right Oka family in a ring $R$. Suppose that $I_{R} \subseteq R$ is such that the right $R$-module $R / I$ has a filtration

$$
0=M_{0} \subseteq M_{1} \subseteq \cdots \subseteq M_{n}=R / I
$$

where each filtration factor is cyclic and of the form $M_{j} / M_{j-1} \cong R / I_{j}$ for some $I_{j} \in \mathcal{F}$. Then $I \in \mathcal{F}$.

An important issue to be dealt with in the proof of Theorem 2.5 is that of similarity of right ideals. Two right ideals $I$ and $J$ of a ring $R$ are similar, written $I \sim J$, if $R / I \cong$ $R / J$ as right $R$-modules. Two results from [33, Section 4] about isomorphic cyclic modules will be relevant to the present paper, the second of which deals directly with similarity of right ideals.

Lemma 2.7 For any ring $R$ with right ideal $I \subseteq R$ and element $a \in R$, the following cyclic right $R$-modules are isomorphic: $R / a^{-1} I \cong(I+a R) / I$.

Proposition 2.8 Every right Oka family $\mathcal{F}$ in a ring $R$ is closed under similarity: if $I \in \mathcal{F}$ then every right ideal similar to $I$ is also in $\mathcal{F}$. 
Finally, in [33, Section 6] we studied a special collection of completely prime right ideals.

Definition 2.9 A module $M \neq 0$ is monoform if, for every nonzero submodule $N_{R} \subseteq$ $M$, every nonzero homomorphism $N \rightarrow M$ is injective. A right ideal $P_{R} \subsetneq R$ is comonoform if the right $R$-module $R / P$ is monoform.

It was shown in [33, Proposition 6.3] that every comonoform right ideal is completely prime. The idea is that comonoform right ideals form an especially "wellbehaved" subset of the completely prime right ideals of any ring.

\section{Point Annihilator Sets for Classes of Modules}

In this section we develop an appropriate notion of a "test set" for certain properties of right ideals in noncommutative rings. This is required for us to state the main theorems along these lines in the next section. Recall that a point annihilator of a module $M_{R}$ is defined to be an annihilator of a nonzero element $m \in M \backslash\{0\}$.

Definition 3.1 Let $\mathcal{C}$ be a class of right modules over a ring $R$. A set $\mathcal{S}$ of right ideals of $R$ is a point annihilator set for $\mathcal{C}$ if every nonzero $M \in \mathcal{C}$ has a point annihilator that lies in $\mathcal{S}$. In addition, we make the following two definitions for special choices of $\mathcal{C}$ :

- A point annihilator set for the class of all right $R$-modules will simply be called a (right) point annihilator set for $R$.

- A point annihilator set for the class of all noetherian right $R$-modules will be called a (right) noetherian point annihilator set for $R$.

Notice that a point annihilator set need not contain the unit ideal $R$, because point annihilators are always proper right ideals. Another immediate observation is that, for a right noetherian ring $R$, a right point annihilator set for $R$ is the same as a right noetherian point annihilator set for $R$.

Remark 3.2 The idea of a point annihilator set $\mathcal{S}$ for a class of modules $\mathcal{C}$ is simply that $\mathcal{S}$ is "large enough" to contain a point annihilator of every nonzero module in $\mathcal{C}$. In particular, our definition does not require every right ideal in $\mathcal{S}$ to actually be a point annihilator for some module in $\mathcal{C}$. This means that any other set $\mathcal{S}^{\prime}$ of right ideals with $\mathcal{S}^{\prime} \supseteq \mathcal{S}$ is also a point annihilator set for $\mathcal{C}$. On the other hand, if $\mathcal{C}_{0} \subseteq \mathcal{C}$ is a subclass of modules, then $\mathcal{S}$ is again a point annihilator set for $\mathcal{C}_{0}$.

Remark 3.3 Notice that $\mathcal{S}$ is a point annihilator set for a class $\mathcal{C}$ of modules iff, for every nonzero module $M_{R} \in \mathcal{C}$, there exists a proper right ideal $I \in \mathcal{S}$ such that the right module $R / I$ embeds into $M$.

The next result shows that noetherian point annihilator sets for a ring $R$ exert a considerable amount of control over the noetherian right $R$-modules. 
Lemma 3.4 $A$ set $\mathcal{S}$ of right ideals in $R$ is a noetherian point annihilator set iff for every noetherian module $M_{R} \neq 0$, there is a finite filtration of $M$

$$
0=M_{0} \subsetneq M_{1} \subsetneq \cdots \subsetneq M_{n}=M
$$

such that, for $1 \leq j \leq n$, there exists $I_{j} \in \mathcal{S}$ such that $M_{j} / M_{j-1} \cong R / I_{j}$.

Proof The "if" direction is easy, so we will prove the "only if" part. For convenience, we will refer to a filtration like the one described above as an $\mathcal{S}$-filtration. Suppose that $\mathcal{S}$ is a noetherian point annihilator set for $R$, and let $M_{R} \neq 0$ be noetherian. We prove by noetherian induction that $M$ has an $\mathcal{S}$-filtration. Consider the set $\mathcal{X}$ of nonzero submodules of $M$ that have an $\mathcal{S}$-filtration. Because $\mathcal{S}$ is a noetherian point annihilator set, it follows that $\mathcal{X}$ is nonempty. Since $M$ is noetherian, $\mathcal{X}$ has a maximal element, say $N$. Assume for contradiction that $N \neq M$. Then $M / N \neq 0$ is noetherian, and by hypothesis there exists $I \in \mathcal{S}$ with $I \neq R$ such that $R / I \cong N^{\prime} / N \subseteq M / N$ for some $N_{R}^{\prime} \subseteq M$. But then $N \subsetneq N^{\prime} \in \mathcal{X}$, contradicting the maximality of $N$. Hence $M=N \in \mathcal{X}$, completing the proof.

We wish to highlight a special type of point annihilator set in the definition below.

Definition 3.5 A set $\mathcal{S}$ of right ideals of a ring $R$ is closed under point annihilators if, for all $I \in \mathcal{S}$, every point annihilator of $R / I$ lies in $\mathcal{S}$. (This is equivalent to saying that $I \in \mathcal{S}$ and $x \in R \backslash I$ imply $x^{-1} I \in \mathcal{S}$.) If $\mathcal{C}$ is a class of right $R$-modules, we will say that $\mathcal{S}$ is a closed point annihilator set for $\mathcal{C}$ if $\mathcal{S}$ is a point annihilator set for $\mathcal{C}$ and $\mathcal{S}$ is closed under point annihilators.

The idea of the above definition is that $\mathcal{S}$ is "closed under passing to further point annihilators of $R / I$ " whenever $I \in \mathcal{S}$. The significance of these closed point annihilator sets is demonstrated by the next result.

Lemma 3.6 Let $\mathcal{C}$ be a class of right modules over a ring $R$ that is closed under taking submodules (e.g. the class of noetherian modules). Suppose that $\mathcal{S}$ is a closed point annihilator set for $\mathcal{C}$. Then for any other point annihilator set $\mathcal{S}_{1}$ of $\mathcal{C}$, the set $\mathcal{S}_{1} \cap \mathcal{S}$ is a point annihilator set for $\mathcal{C}$.

Proof Let $0 \neq M_{R} \in \mathcal{C}$. Because $\mathcal{S}$ is a point annihilator set for $\mathcal{C}$, there exists $0 \neq$ $m \in M$ such that $I:=\operatorname{ann}(m) \in \mathcal{S}$. By the hypothesis on $\mathcal{C}$, the module $m R$ lies in $\mathcal{C}$. Because $\mathcal{S}_{1}$ is also a point annihilator set for $\mathcal{C}$, there exists $0 \neq m r \in m R$ such that $\operatorname{ann}(m r) \in \mathcal{S}_{1}$. The fact that $\mathcal{S}$ is closed implies that ann $(m r) \in \mathcal{S} \cap \mathcal{S}_{1}$. This proves that $\mathcal{S} \cap \mathcal{S}_{1}$ is also a point annihilator set for $\mathcal{C}$.

The prototypical example of a noetherian point annihilator set is the prime spectrum of a commutative ring. In fact, every noetherian point annihilator set in a commutative ring can be "reduced to" some set of prime ideals, as we show below.

Proposition 3.7 In any commutative ring $R$, the set $\operatorname{Spec}(R)$ of prime ideals is a closed noetherian point annihilator set. Moreover, given any noetherian point annihilator set $\mathcal{S}$ for $R$, the set $\mathcal{S} \cap \operatorname{Spec}(R)$ is a noetherian point annihilator subset of $S$ consisting of prime ideals. 
Proof The set $\operatorname{Spec}(R)$ is a noetherian point annihilator set thanks to the standard fact that any noetherian module over a commutative ring has an associated prime; see, for example, [8, Theorem 3.1]. Furthermore, this set is closed because for $P \in \operatorname{Spec}(R)$, the annihilator of any nonzero element of $R / P$ is equal to $P$. The last statement now follows from Lemma 3.6.

In this sense right noetherian point annihilator sets of a ring generalize the concept of the prime spectrum of a commutative ring. However, one should not push this analogy too far: in a commutative ring $R$, any set $\mathcal{S}$ of ideals containing $\operatorname{Spec}(R)$ is also a noetherian point annihilator set! In fact, with the help of Proposition 3.7 it is easy to verify that any commutative ring $R$ has smallest noetherian point annihilator set $\mathcal{S}_{0}:=\{P \in \operatorname{Spec}(R): R / P$ is noetherian $\}$, and that a set $\mathcal{S}$ of ideals of $R$ is a noetherian point annihilator set for $R$ iff $\mathcal{S} \supseteq \mathcal{S}_{0}$.

For most of the remainder of this section, we will record a number of examples of point annihilator sets that will be useful in later applications. Perhaps the easiest example is the following: the family of all right ideals of a ring $R$ is a point annihilator set for any class of right $R$-modules. A less trivial example: the family of maximal right ideals of a ring $R$ is a point annihilator set for the class of right $R$-modules of finite length, or for the larger class of artinian right modules. More specifically, according to Remark 3.3 it suffices to take any set $\left\{\mathfrak{m}_{i}\right\}$ of maximal right ideals such that the $R / \mathfrak{m}_{i}$ exhaust all isomorphism classes of simple right modules.

Example 3.8 Recall that a module $M_{R}$ is said to be semi-artinian if every nonzero factor module of $M$ has nonzero socle, and that a ring $R$ is right semi-artinian if $R_{R}$ is a semi-artinian module. One can readily verify that $R$ is right semi-artinian iff every nonzero right $R$-module has nonzero socle. Thus for such a ring $R$, the set of maximal right ideals is a point annihilator set for $R$, and in particular it is a noetherian point annihilator set for $R$.

Example 3.9 Let $R$ be a left perfect ring, that is, a semilocal ring whose Jacobson radical is left $T$-nilpotent-see [25, Section 23] for details. (Notice that this class of rings includes semiprimary rings, especially right or left artinian rings.) By a theorem of Bass (see [25, (23.20)]), over such a ring, every right $R$-module satisfies DCC on cyclic submodules. Thus every nonzero right module has nonzero socle, and such a ring is right semi-artinian. But $R$ has finitely many simple modules up to isomorphism (because the same is true modulo its Jacobson radical). Choosing a set $\mathcal{S}=\left\{\mathfrak{m}_{1}, \ldots, \mathfrak{m}_{n}\right\}$ of maximal right ideals such that the modules $R / \mathfrak{m}_{i}$ exhaust the isomorphism classes of simple right $R$-modules, we conclude by Remark 3.3 that $\mathcal{S}$ is a point annihilator set for any class of right modules $\mathcal{C}$. Hence $\mathcal{S}$ forms a right noetherian point annihilator set for $R$. (The observant reader will likely have noticed that the same argument applies more generally to any right semi-artinian ring with finitely many isomorphism classes of simple right modules.)

Directly generalizing the fact that the prime spectrum of a commutative ring is a noetherian point annihilator set, we have the following fact, valid for any noncommutative ring. 
Proposition 3.10 The set of completely prime right ideals in any ring $R$ is a noetherian point annihilator set.

Proof Let $M_{R} \neq 0$ be noetherian. For any point annihilator $I=\operatorname{ann}(m)$ with $0 \neq$ $m \in M$, the module $R / I \hookrightarrow M$ is noetherian. Thus $M$ must have a maximal point annihilator $P_{R} \supseteq I$, and $P$ is completely prime by [33, Proposition 5.3].

Recall that in any ring $R$, the set of comonoform right ideals of $R$ forms a subset of the set of all completely prime right ideals of $R$. As we show next, the subset of comonoform right ideals is also a noetherian point annihilator set.

Proposition 3.11 For any ring $R$, the set of comonoform right ideals in $R$ is a closed noetherian point annihilator set.

Proof Because a nonzero submodule of a monoform module is again monoform, Remark 3.3 shows that it is enough to check that any nonzero noetherian module $M_{R}$ has a monoform submodule. This has already been noted, for example, in [30, 4.6.5]. We include a separate proof for the sake of completeness.

Let $L_{R} \subseteq M$ be maximal with respect to the property that there exists a nonzero cyclic submodule $N \subseteq M / L$ that can be embedded in $M$. It is readily verified that $N$ is monoform, and writing $N \cong R / I$ for some comonoform right ideal $I$, the fact that $N$ embeds in $I$ shows that $I$ is a point annihilator of $M$.

Our most "refined" instance of a noetherian point annihilator set for a general noncommutative ring is connected to the concept of (Gabriel-Rentschler) Krull dimension. We review the relevant definitions here, and we refer the reader to the monograph [14] or the textbooks [13, Ch. 15] or [30, Ch. 6] for further details. Define by induction classes $\mathcal{K}_{\alpha}$ of right $R$-modules for each ordinal $\alpha$ (for convenience, we consider -1 to be an ordinal number) as follows. Set $\mathcal{K}_{-1}$ to be the class consisting of the zero module. Then for an ordinal $\alpha \geq 0$ such that $\mathcal{K}_{\beta}$ has been defined for all ordinals $\beta<\alpha$, define $\mathcal{K}_{\alpha}$ to be the class of all modules $M_{R}$ such that, for every descending chain

$$
M_{0} \supseteq M_{1} \supseteq M_{2} \supseteq \cdots
$$

of submodules of $M$ indexed by natural numbers, one has $M_{i} / M_{i+1} \in \bigcup_{\beta<\alpha} \mathcal{K}_{\beta}$ for almost all indices $i$. Now if a module $M_{R}$ belongs to some $\mathcal{K}_{\beta}$, its Krull dimension, denoted $\mathrm{K} \cdot \operatorname{dim}(M)$, is defined to be the least ordinal $\alpha$ such that $M \in \mathcal{K}_{\alpha}$. Otherwise we say that the Krull dimension of $M$ does not exist.

From the definitions it is easy to see that the right $R$-modules of Krull dimension 0 are precisely the (nonzero) artinian modules. Also, a module $M_{R}$ has Krull dimension 1 iff it is not artinian and in every descending chain of submodules of $M$, almost all filtration factors are artinian.

One of the more useful features of the Krull dimension function is that it is an exact dimension function, in the sense that, given an exact sequence $0 \rightarrow L \rightarrow M \rightarrow$ $N \rightarrow 0$ of right $R$-modules, one has

$$
\text { K. } \operatorname{dim}(M)=\sup (\mathrm{K} \cdot \operatorname{dim}(L), \mathrm{K} \cdot \operatorname{dim}(N))
$$


where either side of the equation exists iff the other side exists. See [13, Lemma 15.1] or [30, Lemma 6.2.4] for details.

The Krull dimension can also be used as a dimension measure for rings. We define the right Krull dimension of a ring $R$ to be r. K. $\operatorname{dim}(R)=\mathrm{K} \cdot \operatorname{dim}\left(R_{R}\right)$. The left Krull dimension of $R$ is defined similarly.

Now a module $M_{R}$ is said to be $\alpha$-critical ( $\alpha \geq 0$ an ordinal) if K. $\operatorname{dim}(M)=\alpha$ but $\mathrm{K}$. $\operatorname{dim}(M / N)<\alpha$ for all $0 \neq N_{R} \subseteq M$, and we say that $M_{R}$ is critical if it is $\alpha$-critical for some ordinal $\alpha$. With this notion in place, we define a right ideal $I_{R} \subseteq R$ to be $\alpha$-cocritical if the module $R / I$ is $\alpha$-critical, and we say that $I$ is cocritical if it is $\alpha$ cocritical for some ordinal $\alpha$. Notice immediately that a 0 -critical module is the same as a simple module, and the 0 -cocritical right ideals are precisely the maximal right ideals.

Cocritical right ideals were already studied by A. W. Goldie in [11], though they are referred to there as "critical" right ideals. (The reader should take care not to confuse this terminology with the phrase "critical right ideal" used in a different sense elsewhere in the literature, as mentioned in [33, Section 6].)

Remark 3.12 The first two remarks below are known; for example, see [30, Section 6.2].

(1) Every nonzero submodule $N$ of a critical module $M$ is also critical and has $\mathrm{K} \cdot \operatorname{dim}(N)=\mathrm{K} \cdot \operatorname{dim}(M)$. Suppose that $M$ is $\alpha$-critical. If $\mathrm{K} \cdot \operatorname{dim}(N)<\alpha$, then because $\mathrm{K} \cdot \operatorname{dim}(M / N)<\alpha$, the exactness of Krull dimension would imply the contradiction $\mathrm{K} \cdot \operatorname{dim}(M)<\alpha$. Hence $\mathrm{K} \cdot \operatorname{dim}(N)=\alpha$. Also, for any nonzero submodule $N_{0} \subseteq N$ we have $\mathrm{K} \cdot \operatorname{dim}\left(N / N_{0}\right) \leq \mathrm{K} \cdot \operatorname{dim}\left(M / N_{0}\right)<\alpha$, proving that $N$ is $\alpha$-critical.

(2) A critical module is always monoform. Suppose that $M$ is $\alpha$-critical and fix a nonzero homomorphism $f: C \rightarrow M$ where $C_{R} \subseteq M$. Because $C$ and im $f$ are both nonzero submodules of $M$, they are also $\alpha$-critical by (1). Then $\mathrm{K} \cdot \operatorname{dim}(C)=\mathrm{K} \cdot \operatorname{dim}(\operatorname{im} f)=\mathrm{K} \cdot \operatorname{dim}(C / \operatorname{ker} f)$, so we must have ker $f=0$. Thus $M$ is indeed monoform.

(3) Any cocritical right ideal is comonoform and, in particular, is completely prime. This follows immediately from the preceding remark and the fact [33, Proposition 6.3] that any comonoform right ideal is completely prime.

It is possible to characterize the (two-sided) ideals that are cocritical as right ideals.

Proposition 3.13 For any ring $R$ and any ideal $P \triangleleft R$, the following are equivalent:

(1) $P$ is cocritical as a right ideal;

(2) $R / P$ is a (right Ore) domain with right Krull dimension.

Proof Because every cocritical right ideal is comonoform, (1) $\Longrightarrow(2)$ follows from the fact [33, Proposition 6.5] that a two-sided ideal is comonoform as a right ideal iff its factor ring is a right Ore domain. Because a semiprime ring with right Krull dimension is right Goldie, the two conditions in (2) are equivalent.

$(2) \Longrightarrow(1)$ : It can be shown that, for every module $M_{R}$ whose Krull dimension exists and for every injective endomorphism $f: M \rightarrow M$, one has $\mathrm{K}$. $\operatorname{dim}(M)>$ $\mathrm{K} \cdot \operatorname{dim}(M / f(M))($ see $[13$, Lemma 15.6]). Applying this to $M=S:=R / P$, we see 
that $\mathrm{K} \cdot \operatorname{dim}(S)>\mathrm{K} \cdot \operatorname{dim}(S / x S)$ for all nonzero $x \in S$ (this is also proved in [30, Lemma 6.3.9]). Thus $S_{S}$, and consequently $S_{R}$, are critical modules.

Example 3.14 The last proposition is useful for constructing an ideal of a ring that is (right and left) comonoform but not (right or left) cocritical. If $R$ is a commutative domain that does not have Krull dimension, then the zero ideal of $R$ is prime and thus is comonoform by [33, Proposition 6.5]. But because $R$ does not have Krull dimension, the zero ideal cannot be cocritical by the previous result. For an explicit example, one can take $R=k\left[x_{1}, x_{2}, \ldots\right]$ for some commutative domain $k$. It is shown in [14, Example 10.1] that such a ring does not have Krull dimension, using the fact that a polynomial ring $R[x]$ has right Krull dimension iff the ground ring $R$ is right noetherian.

The reason for our interest in the set of cocritical right ideals is that it is an important example of a noetherian point annihilator set in a general ring.

Proposition 3.15 For any ring $R$, the set of all cocritical right ideals is a closed point annihilator set for the class of right $R$-modules whose Krull dimension exists. In particular, this set is a closed noetherian point annihilator set for $R$.

Proof Because any nonzero module with Krull dimension has a critical submodule (see [13, Lemma 15.8] or [30, Lemma 6.2.10]), Remark 3.3 shows that the set of cocritical right ideals of $R$ is a point annihilator set for the class of right $R$-modules with Krull dimension. Because any noetherian module has Krull dimension (see [13, Lemma 15.3] or [30, Lemma 6.2.3]), we see by Remark 3.2 that this same set is a right noetherian point annihilator set for $R$. The fact that this set is closed under point annihilators follows from Remark 3.12(1).

Let us further examine the relationship between the general noetherian point annihilator sets given in Propositions 3.10, 3.11, and 3.15. From [33, Proposition 6.3] and Remark 3.12(3) we see that there are always the following containment relations (where the first three sets are noetherian point annihilator sets but the last one is not, in general):

$$
\left\{\begin{array}{c}
\text { completely } \\
\text { prime } \\
\text { right ideals }
\end{array}\right\} \supseteq\left\{\begin{array}{c}
\text { comonoform } \\
\text { right ideals }
\end{array}\right\} \supseteq\left\{\begin{array}{c}
\text { cocritical } \\
\text { right ideals }
\end{array}\right\} \supseteq\left\{\begin{array}{c}
\text { maximal } \\
\text { right ideals }
\end{array}\right\} .
$$

Notice that in a commutative ring $R$ the first two sets are equal to $\operatorname{Spec}(R)$ by [33, Corollaries $2.3 \&$ 6.7], and when $R$ is commutative and has Gabriel-Rentschler Krull dimension (e.g., when $R$ is noetherian) the third set is also equal to $\operatorname{Spec}(R)$ by Proposition 3.13. The latter fact provides many examples where the last containment is strict: in any commutative ring $R$ with Krull dimension $>0$ there exists a nonmaximal prime ideal, which must be cocritical. It was shown in [33, Lemma $6.4 \mathrm{ff}$.] and Example 3.14 that the first two inclusions can each be strict. However, the latter example was necessarily non-noetherian. Below we give an example of a noncommutative artinian (hence noetherian) ring over which both containments are strict. This example makes use of the following characterization of semi-artinian 
monoform modules. The proof is straightforward and therefore is omitted. The socle of a module $M_{R}$ is denoted by $\operatorname{soc}(M)$.

Lemma 3.16 Let $M_{R}$ be a semi-artinian $R$-module. Then the following are equivalent:

(1) $M$ is monoform;

(2) For any nonzero submodule $K_{R} \subseteq M$, $\operatorname{soc}(M)$ and $\operatorname{soc}(M / K)$ do not have isomorphic nonzero submodules;

(3) $\operatorname{soc}(M)$ is simple and does not embed into any proper factor module of $M$.

Example 3.17 Let $k$ be a division ring, and let $R$ be the ring of all $3 \times 3$ matrices over $k$ of the form

$$
\left(\begin{array}{lll}
a & b & c \\
0 & d & e \\
0 & 0 & d
\end{array}\right)
$$

One can easily verify (for example, by passing to the factor $R / \operatorname{rad}(R)$ of $R$ by its Jacobson radical) that $R$ has two simple right modules up to isomorphism. We may view these modules as $S_{1}=k$ with right $R$-action given by right multiplication by $a$ in Eq. 3.2 and $S_{2}=k$ with right action given by right multiplication by $d$ in Eq. 3.2. Consider the right ideals

$$
P_{0}:=\left\{\left(\begin{array}{lll}
0 & 0 & 0 \\
0 & d & e \\
0 & 0 & d
\end{array}\right)\right\} \subseteq P_{1}:=\left\{\left(\begin{array}{lll}
0 & 0 & c \\
0 & d & e \\
0 & 0 & d
\end{array}\right)\right\} \subseteq P_{2}:=\left\{\left(\begin{array}{lll}
0 & b & c \\
0 & d & e \\
0 & 0 & d
\end{array}\right)\right\} .
$$

Then the cyclic module $V:=R / P_{0}$ is isomorphic to the space $(k k k)_{R}$ of row vectors with the natural right $R$-action. Notice that $V_{i}:=P_{i} / P_{0}(i=1,2)$ corresponds to the submodule of row vectors whose first $3-i$ entries are zero. One can check that the only submodules of $V$ are $0 \subseteq V_{1} \subseteq V_{2} \subseteq V$, which implies that this is the unique composition series of $V$. It is clear that

$$
V_{1} \cong V_{2} / V_{1} \cong S_{2} \text { and } V / V_{2} \cong S_{1}
$$

We claim that $P_{0}$ is a completely prime right ideal that is not comonoform. To see that it is completely prime, it suffices to show that every nonzero endomorphism of $V=R / P_{0}$ is injective. Indeed, the only proper factors of $V$ are $V / V_{1}$ and $V / V_{2}$. By an inspection of composition factors, neither of these can embed into $V$, proving that $P_{0}$ is completely prime. To see that $P_{0}$ is not comonoform, consider that

$$
\operatorname{soc}(V)=V_{1} \cong V_{2} / V_{1}=\operatorname{soc}\left(V / V_{1}\right) \text {. }
$$

By Lemma 3.16 we see that $R / P_{0}=V$ is not monoform and thus $P_{0}$ is not comonoform.

We also claim that $P_{1}$ is comonoform but not cocritical. Notice that over any right artinian ring, every cyclic critical module has Krull dimension 0. But a 0-critical module is necessarily simple. Thus a cocritical right ideal in a right artinian ring must be maximal. But $P_{1}$ is not maximal and thus is not cocritical. On the other hand, $R / P_{1} \cong V / V_{1}$ has unique composition series $0 \subseteq V_{2} / V_{1} \subseteq V / V_{1}$. This allows us to easily verify, using Lemma 3.16, that $V / V_{1} \cong R / P_{1}$ is monoform, proving that $P_{1}$ is comonoform. 
This same example also demonstrates that the set of completely prime right ideals is not always closed under point annihilators (as in Definition 3.5). This is because the cyclic submodule $V_{2} \subseteq V=R / P_{0}$ certainly has a nonzero noninjective endomorphism, as both of its composition factors are isomorphic.

An example along these lines was already used in [14, p. 11] to show that a monoform module need not be critical. Notice that the completely prime right ideal $P_{0}$ above is such that $R / P_{0}$ is uniform, even if it is not monoform. (This means that the right ideal $P_{0}$ is "meet-irreducible.") An example of a completely prime right ideal whose factor module is not uniform was already given in Example completely prime not meet-irreducible.

Given the containments of noetherian point annihilator sets in Eq. 3.1, one might question the need for the notion of a point annihilator set. Why not simply state all theorems below just for the family of cocritical right ideals? We already have an answer to this question in Example 3.9, which demonstrates that every left perfect ring has a finite right noetherian point annihilator set. The reason we can reduce to a finite set $\mathcal{S}$ in such rings is the fact stated in Remark 3.3 that a noetherian right module only needs to contain a submodule isomorphic to $R / I$ for some $I \in \mathcal{S}$. In other words, $\mathcal{S}$ only needs to contain a single representative from any given similarity class. So while a left perfect ring $R$ may have infinitely many maximal right ideals, it has only finitely many similarity classes of maximal right ideals. Thus we can reduce certain problems about all right ideals of $R$ to a finite set of maximal ideals! This will be demonstrated in Proposition 4.8 and Corollary 5.5, below where we shall prove that a left perfect ring is right noetherian (resp. a PRIR) iff all maximal right ideals belonging to a (properly chosen) finite set are finitely generated (resp. principal).

We have also phrased the discussion in terms of general noetherian point annihilator sets to leave open the possibility of future applications to classes of rings which have nicer noetherian point annihilator sets than the whole set of cocritical right ideals, akin to the class of left perfect rings.

\section{The Point Annihilator Set Theorem}

Having introduced the notion of a point annihilator set, we can now state our fundamental result, the Point Annihilator Set Theorem 4.1. This theorem gives conditions under which one may deduce that one family $\mathcal{F}_{0}$ of right ideals is contained in a second family $\mathcal{F}$ of right ideals. We will most often use it as a sufficient condition for concluding that all right ideals of a ring lie in a particular right Oka family $\mathcal{F}$.

Certain results in commutative algebra state that when every prime ideal in a commutative ring has a certain property, then all ideals in the ring have that property. As mentioned in the introduction, the two motivating examples are Cohen's Theorem 1.1 and Kaplansky's Theorem 1.3. In [29, p. 3017], these theorems were both recovered in the context of Oka families and the Prime Ideal Principle. The useful tool in that context was the "Prime Ideal Principle Supplement" [29, Theorem 2.6]. We have already provided one noncommutative generalization of this tool in the Theorem 2.4, which we used to produce a noncommutative extension of Cohen's Theorem in [33, Theorem 3.8] stating that a ring is right noetherian iff its completely prime right ideals are finitely generated. 
The CPIP Supplement states that for certain right Oka families $\mathcal{F}$, if the set $\mathcal{S}$ of completely prime right ideals lies in $\mathcal{F}$, then all right ideals lie in $\mathcal{F}$. The main goal of this section is to improve upon this result by allowing the set $\mathcal{S}$ to be any point annihilator set. This is achieved in Theorem 4.3 as an application of the Point Annihilator Set Theorem.

The Point Annihilator Set Theorem basically formalizes a general "strategy of proof." For the sake of clarity, we present an informal sketch of this proof strategy before stating the theorem. Suppose that we want to prove that every module with the property $\mathcal{P}$ also has the property $\mathcal{Q}$. Assume for contradiction that there is a counterexample. Use Zorn's Lemma to pass to a counterexample $M$ satisfying $\mathcal{P}$ that is "critical" with respect to not satisfying $\mathcal{Q}$, in the sense that every proper factor module of $M$ satisfies $\mathcal{Q}$ but $M$ itself does not satisfy $\mathcal{Q}$. Argue that $M$ has a nonzero submodule $N$ that satisfies $\mathcal{Q}$. Finally, use the fact that $N$ and $M / N$ have $\mathcal{Q}$ to deduce the contradiction that $M$ has $\mathcal{Q}$.

Our theorem applies in the specific case where one's attention is restricted to cyclic modules. In the outline above, we may think of the properties $\mathcal{P}$ and $\mathcal{Q}$ to be, respectively, " $M=R / I$ where $I \in \mathcal{F}_{0}$ " and " $M=R / I$ where $I \in \mathcal{F}$."

Theorem 4.1 (The Point Annihilator Set Theorem) Let $\mathcal{F}$ be a right Oka family such that every nonempty chain of right ideals in $\mathcal{F}^{\prime}$ (with respect to inclusion) has an upper bound in $\mathcal{F}^{\prime}$.

(1) Let $\mathcal{F}_{0}$ be a semifilter of right ideals in $R$. If $\mathcal{F}$ is a point annihilator set for the class of modules $\left\{R / I: I_{R} \in \operatorname{Max}\left(\mathcal{F}^{\prime}\right) \cap \mathcal{F}_{0}\right\}$, then $\mathcal{F}_{0} \subseteq \mathcal{F}$.

(2) For any right ideal $J_{R} \subseteq R$, if $\mathcal{F}$ is a point annihilator set for the class of modules $R / I$ such that $I \in \operatorname{Max}\left(\mathcal{F}^{\prime}\right)$ and $I \supseteq J$ (resp. $\left.I \supsetneq J\right)$, then all right ideals containing (resp. properly containing) $J$ belong to $\mathcal{F}$.

(3) If $\mathcal{F}$ is a point annihilator set for the class of modules $\left\{R / I: I_{R} \in \operatorname{Max}\left(\mathcal{F}^{\prime}\right)\right\}$, then $\mathcal{F}$ consists of all right ideals of $R$.

Proof Suppose that the hypotheses of (1) hold, and assume for contradiction that there exists $I_{0} \in \mathcal{F}_{0} \backslash \mathcal{F}$. The assumptions on $\mathcal{F}^{\prime}$ allow us to apply Zorn's Lemma to find $I \in \operatorname{Max}\left(\mathcal{F}^{\prime}\right)$ with $I \supseteq I_{0}$. Then $I \in \mathcal{F}_{0}$ because $\mathcal{F}_{0}$ is a semifilter. The point annihilator hypothesis implies that there is a nonzero element $a+I \in R / I$ such that $a^{-1} I=\operatorname{ann}(a+I) \in \mathcal{F}$. On the other hand, $a+I \neq 0+I$ implies that $I+a R \supsetneq I$. By maximality of $I$, this means that $I+a R \in \mathcal{F}$. Because $\mathcal{F}$ is a right Oka family, we arrive at the contradiction $I \in \mathcal{F}$.

Parts (2) and (3) follows from (1) by taking $\mathcal{F}_{0}$ to be, respectively, the set of all right ideals of $R$ (properly) containing $J$ or the set of all right ideals of $R$.

Notice that part (1) above remains true if we weaken the condition on chains in $\mathcal{F}^{\prime}$ to the following: every nonempty chain in $\mathcal{F}^{\prime} \cap \mathcal{F}_{0}$ has an upper bound in $\mathcal{F}^{\prime}$. The latter condition holds if every $I \in \mathcal{F}_{0}$ is such that $R / I$ is a noetherian module, or more generally if $\mathcal{F}_{0}$ satisfies the ascending chain condition (as a partially ordered set with respect to inclusion). However, we shall not make use this observation in the present work.

The following is an illustration of how Theorem 4.1 can be applied in practice. It is well-known that every finitely generated artinian module over a commutative ring has finite length. However, there exist finitely generated (even cyclic) artinian 
right modules over noncommutative rings that do not have finite length; for instance, see [26, Example 4.28]. Here we provide a sufficient condition for all finitely generated artinian right modules over a ring to have finite length.

Proposition 4.2 If all maximal right ideals of a ring $R$ are finitely generated, then every finitely generated artinian right $R$-module has finite length.

Proof It suffices to show that every cyclic artinian right $R$-module has finite length. Let $\mathcal{F}_{0}$ be the semifilter of right ideals $I_{R}$ such that $R / I$ is right artinian, and let $\mathcal{F}$ be the right Oka family of right ideals $I$ such that $R / I$ has finite length. Our goal is then to show that $\mathcal{F}_{0} \subseteq \mathcal{F}$. Because every nonzero cyclic artinian module has a simple submodule, we see that $\mathcal{F}$ is a point annihilator set for the class $\left\{R / I: I \in \mathcal{F}_{0}\right\} \supseteq\left\{R / I: I \in \operatorname{Max}\left(\mathcal{F}^{\prime}\right) \cap \mathcal{F}_{0}\right\}$. To apply Theorem 4.1(1) we will show that every nonempty chain in $\mathcal{F}^{\prime}$ has an upper bound in $\mathcal{F}^{\prime}$. For this, it is enough to check that $\mathcal{F}$ consists of finitely generated right ideals. The hypothesis implies that all simple right $R$-modules are finitely presented. If $I \in \mathcal{F}$ then $R / I$, being a repeated extension of finitely many simple modules, is finitely presented. It follows that $I$ is finitely generated. (The details of the argument that $\mathcal{F}$ consists of f.g. right ideals are in [33, Corollary 4.9].)

In light of the result above, it would be interesting to find a characterization of the rings $R$ over which every finitely generated artinian right $R$-module has finite length. How would such a characterization unite both commutative rings and the rings in which every maximal right ideal is finitely generated?

For our purposes, it will often best to use a variant of the theorem above. This variant keeps with the theme of Cohen's and Kaplansky's results (Theorems 1.1-1.3) of "testing" a property on special sets of right ideals.

Theorem 4.3 Let $\mathcal{F}$ be a right Oka family such that every nonempty chain of right ideals in $\mathcal{F}^{\prime}$ (with respect to inclusion) has an upper bound in $\mathcal{F}^{\prime}$. Let $\mathcal{S}$ be a set of right ideals that is a point annihilator set for the class of modules $\left\{R / I: I_{R} \in \operatorname{Max}\left(\mathcal{F}^{\prime}\right)\right\}$.

(1) Let $\mathcal{F}_{0}$ be a divisible semifilter of right ideals in $R$. If $\mathcal{F}_{0} \cap \mathcal{S} \subseteq \mathcal{F}$, then $\mathcal{F}_{0} \subseteq \mathcal{F}$.

(2) For any ideal $J \triangleleft R$, if all right ideals in $\mathcal{S}$ that contain $J$ belong to $\mathcal{F}$, then every right ideal containing $J$ belongs to $\mathcal{F}$.

(3) If $\mathcal{S} \subseteq \mathcal{F}$, then all right ideals of $R$ belong to $\mathcal{F}$.

Proof As in the previous result, parts (2) and (3) are special cases of part (1). To prove (1), Theorem 4.1 implies that it is enough to show that $\mathcal{F}$ is a point annihilator set for the class of modules $\left\{R / I: I_{R} \in \operatorname{Max}\left(\mathcal{F}^{\prime}\right) \cap \mathcal{F}_{0}\right\}$. Fixing such $R / I$, the hypothesis of part (1) ensures that $R / I$ has a point annihilator in $\mathcal{S}$, say $A=$ ann $(x+I) \in \mathcal{S}$ for some $x+I \in R / I \backslash\{0+I\}$. Because $I \in \mathcal{F}_{0}$ and $\mathcal{F}_{0}$ is divisible, the fact that $A=x^{-1} I$ implies that $A \in \mathcal{F}_{0}$. Thus $A \in \mathcal{S} \cap \mathcal{F}_{0} \subseteq \mathcal{F}$, providing a point annihilator of $R / I$ that lies in $\mathcal{F}$.

We also record a version of Theorem 4.3 adapted especially for families of finitely generated right ideals. Because of its easier formulation, it will allow for simpler proofs as we provide applications of Theorem 4.3. 
Corollary 4.4 Let $\mathcal{F}$ be a right $O$ ka family in a ring $R$ that consists of finitely generated right ideals. Let $\mathcal{S}$ be a noetherian point annihilator set for $R$. Then the following are equivalent:

(1) $\mathcal{F}$ consists of all right ideals of $R$;

(2) $\mathcal{F}$ is a noetherian point annihilator set;

(3) $\mathcal{S} \subseteq \mathcal{F}$.

Proof Given any $I \in \operatorname{Max}\left(\mathcal{F}^{\prime}\right)$, any nonzero submodule of $R / I$ is the image of a right ideal properly containing $I$, which must be finitely generated; thus $R / I$ is a noetherian right $R$-module. Stated another way, the class $\left\{R / I: I \in \operatorname{Max}\left(\mathcal{F}^{\prime}\right)\right\}$ consists of noetherian modules. Thus (1) $\Longleftrightarrow(2)$ follows from Theorem 4.1(3) and (1) $\Longleftrightarrow$ (3) follows from Theorem 4.3(3).

As our first application of the simplified corollary above, we will finally present our noncommutative generalization of Cohen's Theorem 1.1, improving upon [33, Theorem 3.8].

Theorem 4.5 (A noncommutative Cohen's Theorem) Let $R$ be a ring with a right noetherian point annihilator set $\mathcal{S}$. The following are equivalent:

(1) $R$ is right noetherian;

(2) Every right ideal in $\mathcal{S}$ is finitely generated;

(3) Every nonzero noetherian right $R$-module has a finitely generated point annihilator;

(4) Every nonzero noetherian right $R$-module has a nonzero cyclic finitely presented submodule.

In particular, $R$ is right noetherian iff every cocritical right ideal is finitely generated.

Proof The family of finitely generated right ideals is a right Oka family by [33, Proposition 3.7]. The equivalence of (1), (2), and (3) thus follows directly from Corollary 4.4. Also, (3) $\Longleftrightarrow(4)$ comes from the observation that a right ideal $I$ is a point annihilator of a module $M_{R}$ iff there is an injective module homomorphism $R / I \hookrightarrow M$, as well as the fact that $R / I$ is a finitely presented module iff $I$ is a finitely generated right ideal $[24,(4.26)(b)]$. The last statement follows from Proposition 3.15.

In particular, if we take the set $\mathcal{S}$ above to be the completely prime right ideals of $R$, we recover [33, Theorem 3.8]. Our version of Cohen's Theorem will be compared and contrasted with earlier such generalizations in Section 8.

The result above suggests that one might wish to drop the word "cyclic" in characterization (4). This is indeed possible. We present this as a separate result since it does not take advantage of the "formalized proof method" given in Theorem 4.1. However, this result does follow the informal "strategy of proof" outlined at the beginning of this section.

Proposition 4.6 For a ring $R$, the following are equivalent:

(1) $R$ is right noetherian; 
(5) Every nonzero noetherian right $R$-module has a nonzero finitely presented submodule.

Proof Using the numbering from Theorem 4.5, we have (1) $\Longrightarrow(4) \Longrightarrow$ (5). Suppose that (5) holds, and assume for contradiction that there exists a right ideal of $R$ that is not finitely generated. Using Zorn's Lemma, pass to $I_{R} \subseteq R$ that is maximal with respect to not being finitely generated. Then because every right ideal properly containing $I$ is f.g., the module $R / I$ is noetherian. By hypothesis, there is a finitely presented submodule $0 \neq J / I \subseteq R / I$. Then $J \supsetneq I$ implies that $J$ is finitely generated, so that $R / J$ is finitely presented. Because $R / I$ is an extension of the two finitely presented modules $J / I$ and $R / J, R / I$ is finitely presented [28, Example 4.8(2)]. But if $R / I$ is finitely presented then $I_{R}$ is finitely generated [24, (4.26)(b)]. This is a contradiction.

The Akizuki-Cohen Theorem of commutative algebra (cf. [6, pp. 27-28]) states that a commutative ring $R$ is artinian iff it is noetherian and every prime ideal is maximal. Recall that a module $M_{R}$ is finitely cogenerated if any family of submodules of $M$ whose intersection is zero has a finite subfamily whose intersection is zero. In $[29,(5.17)]$ consideration of the class of finitely cogenerated right modules led to the following "artinian version" of Cohen's theorem: a commutative ring $R$ is artinian iff for all $P \in \operatorname{Spec}(R), P$ is finitely generated and $R / P$ is finitely cogenerated. Here we generalize both of these results to the noncommutative setting.

Proposition 4.7 For a ring $R$ with right noetherian point annihilator set $\mathcal{S}$, the following are equivalent:

(1) $R$ is right artinian;

(2) $R$ is right noetherian and for all $P \in \mathcal{S},(R / P)_{R}$ has finite length;

(3) For all $P \in \mathcal{S}, P_{R}$ is finitely generated and $(R / P)_{R}$ has finite length;

(4) For all $P \in \mathcal{S}, P_{R}$ is finitely generated and $(R / P)_{R}$ is finitely cogenerated;

(5) $R$ is right noetherian and every cocritical right ideal of $R$ is maximal;

(6) Every cocritical right ideal of $R$ is finitely generated and maximal.

Proof $(1) \Longleftrightarrow(2) \Longleftrightarrow(3)$ : It is well-known that $R$ is right artinian iff $R_{R}$ has finite length. This equivalence then follows from Corollary 4.4, Theorem 4.5, and the fact that $\mathcal{F}:=\left\{I_{R} \subseteq R: R / I_{R}\right.$ has finite length $\}$ is a right Oka family (see [33, Example 5.18(4)]).

$(1) \Longleftrightarrow(4)$ : It is known that a module $M_{R}$ is artinian iff every quotient of $M$ is finitely cogenerated (see [28, Example 19.0]). Because $\mathcal{F}:=\left\{I_{R} \subseteq R\right.$ : $R / I_{R}$ is finitely cogenerated $\}$ is a right Oka family (see [33, Example 5.18(1B)]), $(1) \Longleftrightarrow$ (4) follows from Corollary 4.4.

We get (1) $\Longleftrightarrow(5) \Longleftrightarrow(6)$ by applying the equivalence of (1), (2), and (3) to the case where $\mathcal{S}$ is the set of cocritical right ideals of $R$, noting that every artinian critical module is necessarily simple.

Of course, the fact that a right noetherian ring is right artinian iff all of its cocritical right ideals are maximal follows from a direct argument involving Krull dimensions of modules. Indeed, given a right noetherian ring $R$ with right Krull dimension $\alpha$, 
choose a right ideal $I \subseteq R$ maximal with respect to $\mathrm{K} \cdot \operatorname{dim}(R / I)=\alpha$. Then for any right ideal $J \supseteq I, \mathrm{~K} \cdot \operatorname{dim}(R / J)<\alpha=\mathrm{K}$. $\operatorname{dim}(R / I)$; hence $I$ is cocritical. So

$$
\text { r. K. } \operatorname{dim}(R)=\sup \left\{\mathrm{K} \cdot \operatorname{dim}(R / I): I_{R} \subseteq R \text { is cocritical }\right\} .
$$

The result now follows once we recall that the 0 -critical modules are precisely the simple modules.

We also mention another noncommutative generalization of the Akizuki-Cohen Theorem due to A. Kertész, which states that a ring $R$ is right artinian iff it is right noetherian and for every prime ideal $P \triangleleft R, R / P$ is right artinian [19]. (We thank the referee for bringing this reference to our attention.)

Another application of Theorem 4.5 tells us when a right semi-artinian ring, especially a left artinian ring, is right artinian. (The definition of a right semi-artinian ring was recalled in Example 3.8.)

\section{Proposition 4.8}

(1) A right semi-artinian ring $R$ is right artinian iff every maximal right ideal of $R$ is finitely generated.

(2) Let $R$ be a left perfect ring (e.g. a semiprimary ring, such as a left artinian ring) and let $\mathfrak{m}_{1}, \ldots, \mathfrak{m}_{n}$ be maximal right ideals such that $R / \mathfrak{m}_{i}$ exhaust all isomorphism classes of simple right modules. Then $R$ is right artinian iff all of the $\mathfrak{m}_{i}$ are finitely generated.

Proof It is easy to check that a right semi-artinian ring $R$ is right artinian iff it is right noetherian. The proposition then follows from Theorem 4.5 and Examples 3.8-3.9.

A result of B. Osofsky [32, Lemma 11] states that a left or right perfect ring $R$ with Jacobson radical $J$ is right artinian iff $J / J^{2}$ is finitely generated as a right $R$-module. This applies, in particular, to left artinian rings. D. V. Huynh characterized which (possibly nonunital) left artinian rings are right artinian in [15, Theorem 1]. In the unital case, his characterization recovers Osofsky's result above for the special class of left artinian rings. We can use our previous result to recover a weaker version of Osofsky's theorem that implies Huynh's result for unital left artinian rings.

Corollary 4.9 Let $R$ be a ring with $J:=\operatorname{rad}(R)$. Then the following are equivalent:

(1) $R$ is right artinian;

(2) $R$ is left perfect and $J$ is a finitely generated right ideal;

(3) $R$ is perfect and $J / J^{2}$ is a finitely generated right $R$-module.

In particular, if $R$ is semiprimary (for instance, if it is left artinian), then $R$ is right artinian iff $\mathrm{J} / \mathrm{J}^{2}$ is finitely generated on the right.

Proof Because any right artinian ring is both perfect and right noetherian, we have $(1) \Longrightarrow(3)$. For (3) $\Longrightarrow(2)$, suppose that $R$ is perfect and that $J / J^{2}$ is right finitely generated. Then for some finitely generated submodule $M_{R} \subseteq J_{R}, J=M+J^{2}$. Since $R$ is right perfect, $J$ is right T-nilpotent. Then by "Nakayama's Lemma" for right Tnilpotent ideals (see $[25,(23.16)]$ ) implies that $J_{R}=M_{R}$ is finitely generated. 
Finally we show (2) $\Longrightarrow(1)$. Suppose that $R$ is left perfect and that $J_{R}$ is finitely generated. For any maximal right ideal $\mathfrak{m}$ of $R$, we have $J \subseteq \mathfrak{m}$. Now $\mathfrak{m} / J$ is a right ideal of the semisimple ring $R / J$ and is therefore finitely generated. Because $J_{R}$ is also finitely generated, we see that $\mathfrak{m}_{R}$ itself is finitely generated. Since this is true for all maximal right ideals of $R$, Proposition 4.8(2) implies that $R$ is right artinian.

Next we give a condition for every finitely generated right module over a ring $R$ to have a finite free resolution (FFR). Notice that such a ring is necessarily right noetherian. Indeed, any module with an FFR is necessarily finitely presented. Thus if every f.g. right $R$-module has an FFR, then for every right ideal $I \subseteq R$ the module $R / I$ must have an FFR and therefore must be finitely presented. It follows (from Schanuel's Lemma [24, (5.1)]) that $I_{R}$ is finitely generated, and $R$ is right noetherian.

Proposition 4.10 Let $\mathcal{S}$ be a right noetherian point annihilator set for a ring $R$ (e.g. the set of cocritical right ideals). Then the following are equivalent.

(1) Every finitely generated right $R$-module has a finite free resolution;

(2) For all $P \in \mathcal{S}, R / P$ has a finite free resolution;

(3) Every right ideal in $\mathcal{S}$ has a finite free resolution.

Proof (1) $\Longrightarrow$ (3): As mentioned before the proposition, if every f.g. right $R$-module has a finite free resolution then $R$ is right noetherian. So every right ideal $I_{R} \subseteq R$ is finitely generated and therefore has a finite free resolution.

Next, (3) $\Longrightarrow$ (2) follows from the easy fact that, given $I_{R} \subseteq R$, if $I$ has a finite free resolution then so does $R / I$. For $(2) \Longrightarrow(1)$, let $\mathcal{F}$ be the family of right ideals $I$ such that $R / I$ has a finite free resolution and assume that $\mathcal{S} \subseteq \mathcal{F}$. This is a right Oka family according to [33, Example 5.12(5)]. Moreover, if $I \in \mathcal{F}$ then $R / I$ is finitely presented. As noted earlier, this implies that $I_{R}$ must be finitely generated [24, (4.26)(b)]. It follows from Corollary 4.4 that every right ideal of $R$ lies in $\mathcal{F}$. Because any finitely generated right $R$-module is an extension of cyclic modules and because the property of having an FFR is preserved by extensions, we conclude that (1) holds.

\section{Families of Principal Right Ideals}

We will use $\mathcal{F}_{\mathrm{pr}}(R)$ to denote the family of principal right ideals of a ring $R$. If the ring $R$ is understood from the context, we may simply use $\mathcal{F}_{\text {pr }}$ to denote this family.

A theorem of Kaplansky [18, Theorem 12.3 \& Footnote 8] states that a commutative ring is a principal ideal ring iff its prime ideals are all principal. In [29, (3.17)] this theorem was recovered via the "PIP supplement." It is therefore reasonable to hope that the methods presented here will lead to a generalization of this result. Specifically, we would like to know whether a ring $R$ is a principal right ideal ring (PRIR) if, say, every cocritical right ideal is principal. It turns out that this is in fact true, but the path to proving the result is not as straightforward as one might imagine. The obvious starting point is to ask whether the family $\mathcal{F}_{\text {pr }}$ of principal right ideals in an arbitrary ring $R$ is a right Oka family. Suppose that $R$ is a ring such that $\mathcal{F}_{\text {pr }}$ is a right Oka family. Then Corollary 4.4 readily applies to $\mathcal{F}_{\text {pr }}$. However, it is not immediately clear whether or not $\mathcal{F}_{\mathrm{pr}}(R)$ is necessarily right Oka for every ring $R$. The following proposition provides some guidance in this matter. 
Proposition 5.1 Let $S \subseteq R$ be a multiplicative set. Then $\mathcal{F}:=\{s R: s \in S\}$ is a right Oka family iff it is closed under similarity. In particular, for any ring $R$, the family $\mathcal{F}_{\mathrm{pr}}$ of principal right ideals is a right Oka family iff it is closed under similarity.

Proof Any right Oka family is closed under similarity by Proposition 2.8. Conversely, assume that the family $\mathcal{F}$ in question is closed under similarity. Suppose that $I+$ $a R, a^{-1} I \in \mathcal{F}$, and write $I+a R=s R$ for some $s \in S$. In the short exact sequence of right $R$-modules

$$
0 \rightarrow \frac{I+a R}{I} \rightarrow \frac{R}{I} \rightarrow \frac{R}{I+a R} \rightarrow 0,
$$

observe that $R /\left(a^{-1} I\right) \cong(I+a R) / I=s R / I \cong R /\left(s^{-1} I\right)$. Because $\mathcal{F}$ is closed under similarity and $a^{-1} I \in \mathcal{F}$, we must also have $s^{-1} I \in \mathcal{F}$. Fix $t \in S$ such that $s^{-1} I=t R$. Then because $I \subseteq I+a R=s R$ we have $I=s\left(s^{-1} I\right)=s t R$, and $s t \in S$ implies that $I \in \mathcal{F}$.

In particular, we have the following "first approximation" to our desired theorem.

Corollary 5.2 Let $\mathcal{S}$ be a right noetherian point annihilator set for $R$. The following are equivalent:

(1) $R$ is a principal right ideal ring;

(2) $\mathcal{F}_{\mathrm{pr}}$ is closed under similarity and every right ideal in $\mathcal{S}$ is principal;

(3) $\mathcal{F}_{\mathrm{pr}}$ is closed under similarity and is a right noetherian point annihilator set.

Proof If $R$ is a PRIR, then $\mathcal{F}_{\text {pr }}$ is equal to the family of all right ideals in $R$ and therefore is closed under similarity. Also, by Proposition 5.1, if $\mathcal{F}_{\text {pr }}$ is closed under similarity then it is a right Oka family. These observations along with Corollary 4.4 establish the equivalence of (1)-(3).

This provides some motivation to explore for which rings the family $\mathcal{F}_{\mathrm{pr}}$ is closed under similarity (and consequently is a right Oka family). Recall that a ring $R$ is called right duo if every right ideal of $R$ is a two-sided ideal. It is easy to see that in any right duo ring, and particularly in any commutative ring, every family of right ideals is closed under similarity. This is because in such a ring $R$, any right ideal $I$ is necessarily a two-sided ideal, so that $I=\operatorname{ann}(R / I)$ can be recovered from the isomorphism class of $R / I$. Thus Proposition 5.1 applies to show that $\mathcal{F}_{\mathrm{pr}}$ is a right Oka family whenever $R$ is a right duo ring, such as a commutative ring. For commutative rings $R$, the fact that $\mathcal{F}_{\text {pr }}$ is an Oka family was already noted in [29, (3.17)].

Another collection of rings in which $\mathcal{F}_{\mathrm{pr}}$ is closed under similarity is the class of local rings. To show that this is the case, we use the fact [33, Proposition 4.6] that a family $\mathcal{F}$ of right ideals of a ring $R$ is closed under similarity iff, for every element $a$ and right ideal $I$ of $R, I+a R=R$ and $a^{-1} I \in \mathcal{F}$ imply $I \in \mathcal{F}$. Suppose that $R$ is local, and that $I_{R} \subseteq R$ and $a \in R$ are such that $I+a R=R$ and $a^{-1} I=x R$ is principal. We want to conclude that $I$ is principal. Write $1=i_{0}+a r$ for some $i_{0} \in I$ and $r \in R$. Let $U(R)$ denote the group of units of $R$. If $i_{0} \in U(R)$, then $I=R$ is principal. Else $i_{0} \notin$ $U(R)$ implies that $1-i_{0}=a r \in U(R)$ and hence $a \in U(R)(R$ local implies that right invertible elements are invertible). But then $a^{-1} I=a^{-1} \cdot I$, so that $I=a\left(a^{-1} I\right)=$ ax $R$ is principal as desired. 
Remark 5.3 In any ring $R$, let $I_{R}, J_{R} \subseteq R$ be right ideals such that $J=x R$ is principal and $R / I \cong R / J$. Then $I$ is generated by at most two elements. To see this, apply Schanuel's Lemma (for instance, see $[24,(5.1)]$ ) to the exact sequences

$$
\begin{aligned}
& 0 \rightarrow I \rightarrow R \rightarrow R / I \rightarrow 0 \text { and } \\
& 0 \rightarrow J \rightarrow R \rightarrow R / J \rightarrow 0
\end{aligned}
$$

to get $R \oplus I \cong R \oplus J$. The latter module is generated by at most two elements. Therefore $I$, being isomorphic to a direct summand of this module, is generated by at most two elements. Thus we see that such $I$ is "not too far" from being principal. (Of course, the same argument shows that if $J_{R} \subseteq R$ is generated by at most $n$ elements and if $I_{R} \subseteq R$ is similar to $J$, then $I$ is generated by at most $n+1$ elements.)

The analysis above also provides the following useful fact: if the module $R_{R}$ is cancellable in the category of (finitely generated) right $R$-modules (or even in the category of finite direct sums of f.g. right ideals), then the family $\mathcal{F}_{\text {pr }}$ is closed under similarity (and hence is a right Oka family). Indeed, if this is the case, suppose that $R / I \cong R / J$ for right ideals $I$ and $J$ with $J$ principal. By the remark above, we have $I$ finitely generated and $R \oplus I \cong R \oplus J$. With the assumption on $R_{R}$ we would have $I_{R} \cong J_{R}$ principal, proving $\mathcal{F}_{\mathrm{pr}}$ to be closed under similarity. (In fact one can similarly show that, over such rings, the minimal number of generators $\mu(I)$ of a f.g. right ideal $I \subseteq R$ is an invariant of the similarity class of $I$.)

This provides another class of rings for which $\mathcal{F}_{\mathrm{pr}}$ is a right Oka family, as follows. Recall that a ring $R$ is said to have (right) stable range 1 if, for $a, b \in R$, $a R+b R=R$ implies that $(a+b r) R=R$ for some $r \in R$ (see [27, Section 1] for details). In [9, Theorem 2] E. G. Evans showed that for any ring with stable range 1, $R_{R}$ is cancellable in the full module category $\mathfrak{M}_{R}$. Thus for any ring $R$ with stable range $1, \mathcal{F}_{\mathrm{pr}}(R)$ is a right Oka family. The class of rings with stable range 1 includes all semilocal rings (see $[25,(20.9)]$ or $[27,(2.10)]$ ), so that this generalizes the case of local rings discussed above.

A similar argument applies in the class of 2-firs. A ring $R$ is said to be a 2-fir (where "fir" stands for "free ideal ring") if the free right $R$-module of rank 2 has invariant basis number and every right ideal of $R$ generated by at most two elements is free. We claim that $\mathcal{F}_{\mathrm{pr}}(R)$ is closed under similarity if $R$ is a 2-fir. Suppose that $I_{R} \subseteq R$ is similar to a principal right ideal $J$. As before, we have $R \oplus I \cong R \oplus J$, and $I$ is generated by at most two elements. So $I \cong R^{m}$, and $J \cong R^{n}$ where $n \leq 1$ because $J$ is principal. Thus $R^{m+1} \cong R^{n+1}$ with $n+1 \leq 2$, and the invariant basis number of the latter free module implies that $m=n \leq 1$. Hence $I_{R} \cong R^{m}$ is a principal right ideal.

There is yet another way in which $\mathcal{F}_{\text {pr }}(R)$ can be closed under similarity. Suppose that every finitely generated right ideal of $R$ is principal; rings satisfying this property are often called right Bezout rings. Then $\mathcal{F}_{\text {pr }}$ is equal to the set of all f.g. right ideals of $R$, which is a right Oka family by [33, Proposition 3.7]. A familiar class of examples of such rings is the class of von Neumann regular rings; in such rings, every finitely generated right ideal is a direct summand of $R_{R}$, and therefore is principal.

We present a summary of the examples above. 
Example 5.4 In each of the following types of rings, the family $\mathcal{F}_{\mathrm{pr}}$ is closed under similarity and thus is a right Oka family:

(1) Right duo rings (including commutative rings);

(2) Rings with stable range 1 (including semilocal rings);

(3) 2-firs;

(4) Right Bezout rings (including von Neumann regular rings).

One collection of semilocal rings that we have already mentioned is the class of left perfect rings. An application of Corollary 5.2 in this case gives the following.

Corollary 5.5 Let $R$ be a left perfect ring (e.g. a semiprimary ring, such as a onesided artinian ring), and let $\mathfrak{m}_{1}, \ldots, \mathfrak{m}_{n} \subseteq R$ be maximal right ideals such that the $R / \mathfrak{m}_{i}$ represent all isomorphism classes of simple right $R$-modules. Then $R$ is a PRIR iff all of the $\mathfrak{m}_{i}$ are principal right ideals.

Proof By Example 5.4(2), $\mathcal{F}_{\mathrm{pr}}$ is an Oka family of right ideals in $R$. By Example 3.9, the set $\left\{\mathfrak{m}_{i}\right\}$ is a right noetherian point annihilator set. The claim then follows from Corollary 5.2.

As it turns out, the family $\mathcal{F}_{\text {pr }}$ can indeed fail to be right Oka, even in a noetherian domain! This will be shown in Example 5.7 below, with the help of the following lemma.

Lemma 5.6 Let $R$ be a ring with an element $x \in R$ that is not a left zero-divisor.

(A) If $J$ and $K$ are right ideals of $R$ with $J \subseteq x R$, then

$$
x^{-1}(J+K)=x^{-1} J+x^{-1} K .
$$

(B) For any $f \in R$,

$$
x^{-1}(x f R+(1+x y) R)=f R+(1+y x) R .
$$

Proof

(A) The containment " $\supseteq$ " holds without any assumptions on $x, J$, or $K$ because $x\left(x^{-1} J+x^{-1} K\right)=x \cdot\left(x^{-1} J\right)+x \cdot\left(x^{-1} K\right) \subseteq J+K$. To show " $\subseteq$ " let $f \in$ $x^{-1}(J+K)$, so that there exist $j \in J$ and $k \in K$ such that $x f=j+k$. Because $J \subseteq x R$, there exists $j_{0}$ such that $j=x j_{0}$; notice that $j_{0} \in x^{-1} J$. Then we have $k=x k_{0}$ for $k_{0}=f-j_{0} \in x^{-1} K$. Now $x f=x j_{0}+x k_{0}$, and because $x$ is not a left zero-divisor we have $f=j_{0}+k_{0} \in x^{-1} J+x^{-1} K$.

(B) Setting $J=x f R$ and $K=(1+x y) R$, one may compute that $x^{-1} J=f R$ and $x^{-1} K=(1+y x) R$ (using the fact that $x$ is not a left zero divisor). The claim follows directly from part (A).

Example $5.7 A$ ring in which $\mathcal{F}_{\mathrm{pr}}$ is not a right Oka family. Let $k$ be a field and let $R:=A_{1}(k)=k\langle x, y: x y=y x+1\rangle$ be the first Weyl algebra over $k$. Then $R$ is 
known to be a noetherian domain (which is simple if $k$ has characteristic 0). Define the right ideal

$$
I_{R}:=x^{2} R+(1+x y) R \subseteq R,
$$

which is shown to be nonprincipal in [30,7.11.8]. Because $I+x R$ contains both $1+$ $x y \in I$ and $x y \in x R$, we must have $1 \in I+x R=R$.

Because $1+y x=x y \in x R$, Lemma 5.6(B) above (with $f=x$ ) implies that $x^{-1} I=$ $x R+(1+y x) R=x R$. Therefore we have $I+x R=R$ and $x^{-1} I=x R$ both members of $\mathcal{F}_{\mathrm{pr}}$ with $I \notin \mathcal{F}_{\mathrm{pr}}$ proving that $\mathcal{F}_{\mathrm{pr}}$ is not a right Oka family. In fact we have $R / I \cong R / x R$ where $I$ is not principal (the isomorphism follows from Lemma 2.7), showing explicitly that $\mathcal{F}_{\text {pr }}$ is not closed under similarity as predicted by Proposition 5.1. In agreement with Remark 5.3, $I$ is generated by two elements.

Notice that $R / x R \cong k[y]$, where $k[y] \subseteq R$ acts by right multiplication and $x \in R$ acts as $-\partial / \partial y$. If $k$ has characteristic 0 then this module is evidently simple, and because $R / I \cong R / x R$ we see that $I$ is a maximal right ideal. If instead $\operatorname{char}(k)=p>$ 0 , then $R / x R \cong k[y]$ is evidently not simple, and not even artinian (the submodules $y^{n p} k[y]$ form a strictly descending chain for $n \geq 0$ ). But every proper factor of this module has finite dimension over $k$ and is therefore artinian. So we see that $R / I \cong R / x R$ is 1 -critical, making $I$ a 1 -cocritical right ideal. Thus regardless of the characteristic of $k$, the nonprincipal right ideal $I$ is cocritical.

On the other hand, when char $k=0$ the ring $\mathbb{M}_{2}(R)$ is known to be a principal (right and left) ideal ring-see [30, 7.11.7]. Then $\mathcal{F}_{\mathrm{pr}}\left(\mathbb{M}_{2}(R)\right)$ is equal to the set of all right ideals in $\mathbb{M}_{2}(R)$ and thus is a right Oka family. So we see that the property " $\mathcal{F}_{\mathrm{pr}}(R)$ is a right Oka family" is not Morita invariant.

It would be very desirable to eliminate the condition in Corollary 5.2 that $\mathcal{F}_{\text {pr }}$ is closed under similarity. It turns out that a suitable strengthening of the hypothesis on the point annihilator set $\mathcal{S}$ will in fact allow us to discard that assumption. The following constructions will help us achieve this goal in Theorem 5.11 below. Recall that for right ideals $I$ and $J$ of a ring $R$, we write $I \sim J$ to mean that $I$ and $J$ are similar.

Definition 5.8 For any ring $R$, we define

$$
\begin{aligned}
\mathcal{F}_{\mathrm{pr}}^{\circ}(R) & :=\left\{I_{R} \subseteq R: \forall J_{R} \subseteq R, I \sim J \Longrightarrow J \in \mathcal{F}_{\mathrm{pr}}(R)\right\} \\
& =\left\{I_{R} \subseteq R: I \text { is only similar to principal right ideals }\right\}
\end{aligned}
$$

Alternatively, $\mathcal{F}_{\mathrm{pr}}^{\circ}(R)$ is the largest subset of $\mathcal{F}_{\mathrm{pr}}(R)$ that is closed under similarity.

As with $\mathcal{F}_{\text {pr }}$, we will often write $\mathcal{F}_{\mathrm{pr}}^{\circ}$ in place of $\mathcal{F}_{\mathrm{pr}}^{\circ}(R)$ when the ring $R$ is understood from the context. We saw in Proposition 5.1 that certain families of principal right ideals are right Oka precisely when they are closed under similarity. But $\mathcal{F}_{\text {pr }}^{\circ}$ is the largest family of principal right ideas that is closed under similarity. Thus one might wonder whether $\mathcal{F}_{\mathrm{pr}}^{\circ}$ is a right Oka family. As it turns out, we are very fortunate and this is in fact true in every ring!

Proposition 5.9 For any ring $R, \mathcal{F}_{\mathrm{pr}}^{\circ}(R)$ is an Oka family of right ideals. 
Proof We will denote $\mathcal{F}:=\mathcal{F}_{\mathrm{pr}}^{\circ}(R)$. Because $I_{R} \sim R_{R}$ implies $I=R \in \mathcal{F}_{\mathrm{pr}}$, we see that $R \in \mathcal{F}$. Suppose that $I_{R} \subseteq R$ and $a \in R$ are such that $I+a R, a^{-1} I \in \mathcal{F}$. Set $C_{1}:=R / a^{-1} I$ and $C_{2}:=R /(I+a R)$, so that we have an exact sequence

$$
0 \rightarrow C_{1} \rightarrow R / I \rightarrow C_{2} \rightarrow 0 .
$$

To prove that $I \in \mathcal{F}$, let $J_{R} \subseteq R$ be such that $R / J \cong R / I$. We need to show that $J$ is principal. There is also an exact sequence

$$
0 \rightarrow C_{1} \rightarrow R / J \rightarrow C_{2} \rightarrow 0 \text {. }
$$

Thus there exists $x \in R$ with $C_{1} \cong(J+x R) / J$ and $C_{2} \cong R /(J+x R)$. But then $R /(I+a R)=C_{2} \cong R /(J+x R)$ and $I+a R \in \mathcal{F}$ imply that $J+x R=c R$ for some $c \in R$. Now

$$
\frac{R}{a^{-1} I}=C_{1} \cong \frac{J+x R}{J}=\frac{c R}{J} \cong \frac{R}{c^{-1} J}
$$

and $a^{-1} I \in \mathcal{F}$, so we find that $c^{-1} J$ is principal. Then $J \subseteq J+x R=c R$ gives $J=$ $c\left(c^{-1} J\right)$, proving that $J$ is principal.

The following elementary observation will be useful in a number of places. It is simply a convenient restatement of the fact that $\mathcal{F}_{\mathrm{pr}}^{\circ}$ is the largest set of principal right ideals that is closed under similarity.

Lemma 5.10 Let $\mathcal{S}$ be a set of right ideals of a ring $R$ that is closed under similarity. If $\mathcal{S} \subseteq \mathcal{F}_{\mathrm{pr}}$, then $\mathcal{S} \subseteq \mathcal{F}_{\mathrm{pr}}^{\circ}$ (and, of course, conversely).

We are finally ready to state and prove our noncommutative generalization of the Kaplansky-Cohen Theorem 1.3.

Theorem 5.11 (A noncommutative Kaplansky-Cohen Theorem) For any ring $R$, let $\mathcal{S}$ be a right noetherian point annihilator set that is closed under similarity. The following are equivalent:

(1) $R$ is a principal right ideal ring;

(2) Every right ideal in $\mathcal{S}$ is principal;

(3) $\mathcal{F}_{\mathrm{pr}}^{\circ}$ is a right noetherian point annihilator set.

In particular, $R$ is a principal right ideal ring iff every cocritical right ideal of $R$ is principal.

Proof The set of cocritical right ideals of $R$ is a noetherian point annihilator set that is closed under similarity, so it suffices to prove the equivalence of (1)-(3). It is easy to see that (1) is equivalent to the claim that all right ideals lie in $\mathcal{F}_{\mathrm{pr}}^{\circ}$. Also, it follows from Lemma 5.10 that (2) holds precisely when $\mathcal{S} \subseteq \mathcal{F}_{\mathrm{pr}}^{\circ}$. The equivalence of (1)-(3) now follows from Corollary 4.4 and Proposition 5.9.

As with Cohen's Theorem, there exist previous noncommutative generalizations of the Kaplansky-Cohen theorem in the literature. In Section 8 we relate our theorem with these earlier results. 
Comparing our two versions of the Kaplansky-Cohen Theorem, we see that Corollary 5.2 follows from Theorem 5.11, at least if we consider condition (3) in each equivalence. (Recall Remark 3.2, and the fact that $\mathcal{F}_{\mathrm{pr}}^{\circ} \subseteq \mathcal{F}_{\mathrm{pr}}$.) However, this does not mean that Corollary 5.2 is obsolete. It is clear that Theorem 5.11 is preferable to Corollary 5.2 if we have enough knowledge about the point annihilator set $\mathcal{S}$ but we do not know whether the family $\mathcal{F}_{\text {pr }}$ is closed under similarity. On the other hand, if we are working in a class of rings for which we know that $\mathcal{F}_{\text {pr }}$ is closed under similarity, then Corollary 5.2 may be of more use. This proved to be the case in Corollary 5.5, where we were able to reduce the point annihilator set $\mathcal{S}$ to a finite set.

Notice that our earlier examination of the Weyl algebra $A_{1}(k)$ in Example 5.7 fits nicely with Theorem 5.11, because the nonprincipal right ideal discussed in that example was shown to be cocritical.

As a simple application of Theorem 5.11, we can show that a domain $R$ with right Krull dimension $\leq 1$ is a principal right ideal domain iff its maximal right ideals are principal. Indeed, by Proposition 3.13 the zero ideal of $R$ is 1 -cocritical as a right ideal (and it is, of course, principal). Thus any nonzero cocritical right ideal of $R$ is 0 -critical and therefore is a maximal right ideal. The claim then follows from Theorem 5.11. However, we will prove a substantially more general version of this fact in Proposition 7.1.

\section{Families Closed Under Direct Summands}

In this section we will develop further generalizations of Cohen's Theorem and the Kaplansky-Cohen theorems by further reducing the set of right ideals in a ring which we are required to "test." In particular, where our previous theorems stated that it was sufficient to check that every right ideal in some noetherian point annihilator set $\mathcal{S}$ is finitely generated (or principal), we will further reduce the task to checking that every essential right ideal in $\mathcal{S}$ is finitely generated (or principal). We begin with a definition, temporarily digressing to families of submodules of a given module other than $R_{R}$.

Definition 6.1 Let $M_{R}$ be a module over a ring $R$. We will say that a family $\mathcal{F}$ of submodules of $M$ is closed under direct summands if for any $N \in \mathcal{F}$, any direct summand of $N$ also lies in $\mathcal{F}$.

Notice that a family $\mathcal{F}$ of submodules of $M$ that is closed under direct summands necessarily has $0 \in \mathcal{F}$ as long as $\mathcal{F} \neq \varnothing$. The following result is the reason for our interest in families that are closed under direct summands. It shows the link between such families and the essential submodules of $M$.

Lemma 6.2 In a module $M_{R}$, let $\mathcal{F}$ be a family of submodules that is closed under direct summands. Then all submodules of $M$ lie in $\mathcal{F}$ iff all essential submodules of $M$ lie in $\mathcal{F}$. In particular, if $\mathcal{F}$ is a family of right ideals in a ring $R$ that is closed under direct summands, then all right ideals of $R$ lie in $\mathcal{F}$ iff all essential right ideals of $R$ lie in $\mathcal{F}$. 
Proof ("If" direction) Suppose that every essential submodule of $M$ lies in $\mathcal{F}$, and let $L_{R} \subseteq M$. By Zorn's lemma there exists a submodule $N_{R}$ maximal with respect to $L \cap N=0$ (in the literature, such $N$ is referred to as a complement to $L$ ). We claim that $N \oplus L$ is an essential submodule of $M$. Indeed, assume for contradiction that $0 \neq K \subseteq M$ is a submodule such that $(L \oplus N) \cap K=0$. Then we have the direct sum $L \oplus N \oplus K$ in $M$. It follows that $L \cap(N \oplus K)=0$, contradicting the maximality of $N$.

By assumption, $N \oplus L \subseteq_{e} M$ implies that $N \oplus L \in \mathcal{F}$. Then because $\mathcal{F}$ is closed under direct summands, we conclude that $N \in \mathcal{F}$.

With this result as our motivation, let us consider a few examples of families of right ideals that are closed under direct summands.

Example 6.3 In any module $M_{R}$, the easiest nontrivial example of a family that is closed under direct summands is the family $\mathcal{F}$ of all direct summands of $M$ ! The application of Lemma 6.2 in this case says that a module $M$ is semisimple iff every essential submodule of $M$ is a direct summand. However, it is easy to check that a direct summand of $M$ is essential in $M$ iff it is equal to $M$. So this says that a module is semisimple iff it has no proper essential submodules. This is a known result; for instance, see [28, Example 3.9].

Example 6.4 The family of finitely generated submodules of a module $M_{R}$ is certainly closed under direct summands. It follows that a module $M$ is right noetherian iff all of its essential submodules are finitely generated. Again, this fact can be found, for instance, in [28, Example 6.11].

We can generalize the result above as follows. Let $\alpha$ be any cardinal (finite or infinite), and let $\mathcal{F}$ be the family of all submodules of $M$ that have a generating set of size $<\alpha$. Then $\mathcal{F}$ is again closed under direct summands. So every submodule of $M$ is generated by $<\alpha$ elements iff the essential submodules of $M$ are all generated by $<\alpha$ elements.

Taking $M_{R}=R_{R}$ and $\alpha=2$, we see in particular that $\mathcal{F}_{\mathrm{pr}}$ is closed under direct summands, and Lemma 6.2 implies that $R$ is a PRIR iff its essential right ideals are principal.

Here we end our digression into families of submodules of arbitrary modules and focus our attention on families of right ideals in a ring $R$ that are closed under direct summands. The next two examples are of a homological nature.

Example 6.5 For a module $M_{R}$, let $\mathcal{F}$ be the family of right ideals $I \subseteq R$ such that every module homomorphism $f: I \rightarrow M$ extends to a homomorphism $R \rightarrow M$. This was shown to be a right Oka family in [33, Proposition 5.16]. We claim that $\mathcal{F}$ is closed under direct summands. For if $I \oplus J \in \mathcal{F}$ and $f: I \rightarrow M$ is any homomorphism, then we may extend $f$ trivially to $I \oplus J \rightarrow M$. This morphism in turn extends to $R \rightarrow M$ because $I \oplus J \in \mathcal{F}$. Hence $I \in \mathcal{F}$.

By Baer's Criterion, every right ideal lies in $\mathcal{F}$ precisely when $M$ is injective. So applying Lemma 6.2, we find that $M$ is injective iff every essential right ideal of $R$ lies in $\mathcal{F}$. This "essential version" of Baer's Criterion has been noticed before; for instance, see [28, Example 3.26]. 
More generally, for any module $M_{R}$ and integer $n \geq 0$, let $\mathcal{F}_{M}^{n}$ denote the family of right ideals $I \subseteq R$ such that $\operatorname{Ext}_{R}^{n+1}(R / I, M)=0$. The family $\mathcal{F}$ above was shown to be equal to $\mathcal{F}_{M}^{0}$ in the proof of [33, Proposition 5.16]. We claim that the families $\mathcal{F}^{n}$ are closed under direct summands. The case $n=0$ is covered above, so suppose that $n \geq 1$. Note that $\operatorname{Ext}_{R}^{n}(R, M)=\operatorname{Ext}_{R}^{n+1}(R, M)=0$ because $R_{R}$ is projective. So for any right ideal $K \subseteq R$, the long exact sequence in Ext provides isomorphisms $\operatorname{Ext}_{R}^{n}(K, M) \cong \operatorname{Ext}_{R}^{n+1}(R / K, M)$. Thus for any direct sum of right ideals $I \oplus J \subseteq R$, combining this observation with a standard fact about Ext and direct sums gives

$$
\begin{aligned}
\operatorname{Ext}_{R}^{n+1}(R /(I \oplus J), M) & \cong \operatorname{Ext}_{R}^{n}(I \oplus J, M) \\
& \cong \operatorname{Ext}_{R}^{n}(I, M) \oplus \operatorname{Ext}_{R}^{n}(J, M) \\
& \cong \operatorname{Ext}_{R}^{n+1}(R / I, M) \oplus \operatorname{Ext}_{R}^{n+1}(R / J, M)
\end{aligned}
$$

This makes it clear that if $I \oplus J \in \mathcal{F}_{M}^{n}$, then $I \in \mathcal{F}_{M}^{n}$.

Extending Baer's Criterion, one can show that a module $M_{R}$ has injective dimension $\leq n$ iff $\operatorname{Ext}_{R}^{n+1}(R / I, M)=0$ for all right ideals $I$ of $R$ (this is demonstrated in the proof of [36, Theorem 8.16]). If we apply Lemma 6.2 to the family $\mathcal{F}_{M}^{n}$, we see that for any module $M_{R}$ we have $\operatorname{id}(M) \leq n$ iff $\operatorname{Ext}_{R}^{n+1}(R / I, M)=0$ for all essential right ideals $I$ of $R$.

Example 6.6 As an application of Example 6.5 above, we produce another example of a family that is closed under direct summands. Let $\mathcal{F}^{n}$ be the family of all right ideals of $R$ such that $\operatorname{pd}(R / I) \leq n$. Because $R / I$ has projective dimension $\leq n$ iff $\operatorname{Ext}_{R}^{n+1}(R / I, M)=0$ for all modules $M$, we see that $\mathcal{F}^{n}$ is equal to the intersection of all of the families $\mathcal{F}_{M}^{n}$ as $M$ ranges over all right $R$-modules. Since all of these families are closed under direct summands, $\mathcal{F}^{n}$ is also closed under summands. In this case we can apply Lemma 6.2 to say that a ring $R$ has r. $\operatorname{gl} \operatorname{dim}(R) \leq n$ iff $\operatorname{pd}(R / I) \leq n$ for all essential right ideals $I_{R} \subseteq R$. Notice that when $n=0, \mathcal{F}^{0}$ is the family of right ideal direct summands mentioned in Example 6.3.

Before continuing to the heart of this section, we require a small observation as well as a new definition.

Remark 6.7 Notice that the set of essential right ideals is a divisible semifilter, and is closed under similarity. It is easy to see that the set is a semifilter. To see that it is divisible, we will use the following fact about essential submodules: for any homomorphism of modules $f: M_{R} \rightarrow N_{R}$ and any essential submodule $N_{0} \subseteq N$, the preimage $f^{-1}\left(N_{0}\right)$ is an essential submodule of $M$ (see [28, Example 3.7] for a proof of this fact). Now given a right ideal $I \subseteq R, x^{-1} I$ is the preimage of the right ideal $I$ under the homomorphism $R_{R} \rightarrow R_{R}$ given by left multiplication by $x$. Thus if $I$ is an essential right ideal, so is $x^{-1} I$. Finally, to see that this set is closed under similarity, one only needs to realize that $I_{R} \subseteq R$ is essential iff $R / I$ is a singular module; see [28, Example 2(b)].

Definition 6.8 Let $\mathcal{F}$ be a family of right ideals in a ring $R$. We define

$$
\widetilde{\mathcal{F}}:=\left\{I_{R} \subseteq R: I \oplus J \in \mathcal{F} \text { for some } J_{R} \subseteq R\right\} .
$$


This is the smallest family of right ideals containing $\mathcal{F}$ that is closed under direct summands.

The next result, which is fundamental to this section, is a variation of Theorem 4.3 and Corollary 4.4.

Theorem 6.9 Let $\mathcal{F}$ be an Oka family of right ideals in a ring $R$.

(1) Assume that every chain of right ideals in $\mathcal{F}^{\prime}$ has an upper bound in $\mathcal{F}^{\prime}$, and let $\mathcal{S}$ be a point annihilator set for the class of modules $\left\{R / I: I \in \operatorname{Max}\left(\mathcal{F}^{\prime}\right)\right\}$. If every essential right ideal in $\mathcal{S}$ lies in $\mathcal{F}$, then all right ideals of $R$ lie in $\widetilde{\mathcal{F}}$.

(2) Let $\mathcal{S}$ be a noetherian point annihilator set for $R$, and assume that $\mathcal{F}$ consists of finitely generated right ideals. If every essential right ideal in $\mathcal{S}$ lies in $\mathcal{F}$, then all right ideals of $R$ lie in $\widetilde{\mathcal{F}}$.

Proof To prove (1), let $\mathcal{S}$ and $\mathcal{F}$ satisfy the given hypotheses. Let $\mathcal{F}_{0}$ denote the divisible semifilter of essential right ideals of $R$. By assumption we have $\mathcal{F}_{0} \cap \mathcal{S} \subseteq \mathcal{F}$, so it follows from Theorem 4.3 that $\mathcal{F}_{0} \subseteq \mathcal{F}$. Then all essential right ideals of $R$ lie in $\widetilde{\mathcal{F}} \supseteq \mathcal{F}$, and it follows from Lemma 6.2 that all right ideals lie in $\widetilde{\mathcal{F}}$.

Now (2) follows from (1) because the fact that $\mathcal{F}$ consists of finitely generated right ideals implies both that every chain of right ideals in $\mathcal{F}^{\prime}$ has an upper bound in $\mathcal{F}^{\prime}$ and that the class $\left\{R / I: I \in \operatorname{Max}\left(\mathcal{F}^{\prime}\right)\right\}$ consists of noetherian modules (as in the proof of Corollary 4.4).

In particular, if the right $\underset{\widetilde{F}}{ } \mathrm{Oka}$ family $\mathcal{F}$ in the theorem above is in fact closed under direct summands, then $\widetilde{\mathcal{F}}=\mathcal{F}$. Thus in this case Theorem 6.9 is a generalization of Theorem 4.3. Our first application of this result will be a strengthening of the noncommutative Cohen's Theorem 4.5.

Theorem 6.10 For a ring $R$, let $\mathcal{S}$ be a right noetherian point annihilator set (such as the set of cocritical right ideals). Then $R$ is right noetherian iff every essential right ideal in $\mathcal{S}$ is finitely generated.

Proof ("If" direction) This follows directly from Example 6.4 and Theorem 6.9(2) by taking $\mathcal{F}=\widetilde{\mathcal{F}}$ to be the family of finitely generated right ideals of $R$.

Our next application of Theorem 6.9 will strengthen our noncommutative version of the Kaplansky-Cohen Theorem 5.11. The careful statement of Theorem 6.9 will pay off here.

Theorem 6.11 Let $R$ be a ring with noetherian point annihilator set $\mathcal{S}$ that is closed under similarity (such as the set of cocritical right ideals). Then $R$ is a principal right ideal ring iff every essential right ideal in $\mathcal{S}$ is principal.

Proof ("If" direction) Suppose that every essential right ideal in $\mathcal{S}$ is principal, and set $\mathcal{F}:=\mathcal{F}_{\text {pr }}^{\circ}$. If $\mathcal{S}_{0} \subseteq \mathcal{S}$ is the set of essential right ideals in $\mathcal{S}$, then $\mathcal{S}_{0}$ is closed under similarity because both $\mathcal{S}$ and the set of essential right ideals are closed under similarity (recall Remark 6.7). By hypothesis $\mathcal{S}_{0} \subseteq \mathcal{F}_{\text {pr }}$, so Lemma 5.10 gives $\mathcal{S}_{0} \subseteq \mathcal{F}_{\text {pr }}^{\circ}=: \mathcal{F}$. That is, every essential right ideal in $\mathcal{S}$ lies in $\mathcal{F}$. Now Theorem 6.9(2) 
implies that all right ideals of $R$ lie in $\widetilde{\mathcal{F}}$. But $\mathcal{F}_{\text {pr }}$ is closed under direct summands by Example 6.4 , so $\mathcal{F} \subseteq \mathcal{F}_{\text {pr }}$ implies that $\widetilde{\mathcal{F}} \subseteq \mathcal{F}_{\text {pr }}$. Hence every right ideal of $R$ is principal.

Our final applications of Theorem 6.9 show how to reduce the test sets for various homological properties in a right noetherian ring.

Theorem 6.12 Let $R$ be a right noetherian ring, and let $\mathcal{S}$ be a right (noetherian) point annihilator set for $R$ (such as the set of cocritical right ideals).

(1) A module $M_{R}$ has injective dimension $\leq n$ iff $\operatorname{Ext}_{R}^{n+1}(R / P, M)=0$ for all essential right ideals $P \in \mathcal{S}$.

(2) Every finitely generated right $R$-module has finite projective dimension iff, for every essential right ideal $P \in \mathcal{S}$, one has $\operatorname{pd}(R / P)<\infty$.

(3) r. $\operatorname{gl} \operatorname{dim}(R)=\sup \{\operatorname{pd}(R / P): P \in \mathcal{S}$ is an essential right ideal $\}$.

Proof For a module $M_{R}$ and a nonnegative integer $n$, let $\mathcal{F}_{M}^{n}$ and $\mathcal{F}^{n}$ be the families introduced in Examples 6.5 and 6.6, where they were shown to be closed under direct summands. These families were shown to be right Oka families in [33, Section 5.B]. Defining $\mathcal{F}^{\infty}:=\bigcup_{n=1}^{\infty} \mathcal{F}^{n}$, it follows that $\mathcal{F}^{\infty}$ is also a right Oka family that is closed under direct summands.

For part (1), we note that a module $M_{R}$ has injective dimension $\leq n$ iff $\mathcal{F}_{M}^{n}$ consists of all right ideals of $R$, which happens iff all essential right ideals in $\mathcal{S}$ lie in $\mathcal{F}_{M}^{n}$ according to Theorem 6.9(2). Next we prove part (2). Because every finitely generated right $R$-module is has a finite filtration with cyclic filtration factors, and because the finiteness of projective dimension is preserved by extensions, we see that every finitely generated right $R$-module has finite projective dimension iff every cyclic right $R$-module does, iff $\mathcal{F}^{\infty}$ consists of all right ideals. By Theorem 6.9, this occurs iff all essential right ideals in $\mathcal{S}$ lie in $\mathcal{F}^{\infty}$.

Part (3) similarly follows from Theorem 6.9 applied to the family $\mathcal{F}^{n}$, noting that $R$ has right global dimension $\leq n$ iff $\mathcal{F}^{n}$ consists of all right ideals.

The above joins a whole host of results stating that certain homological properties can be tested on special sets of ideals. We mention only a few relevant references here. When $R$ is commutative, $\mathcal{S}=\operatorname{Spec}(R)$, and $n=0$ in part (1), the theorem above recovers a result of J.A. Beachy and W.D. Weakley in [2]. Part (2) generalizes a result characterizing commutative regular rings, the "globalizations" of regular local rings (see $[24,(5.94)])$. Many results along the lines of part (3) are known. For instance, a result of J. J. Koker in [22, Lemma 2.1] implies that if a ring $R$ has right Krull dimension, then its right global dimension is equal to the supremum of the projective dimensions of the right modules $R / P$, where $P$ ranges over the cocritical right ideals of $R$. On the other hand, for a commutative noetherian ring $R$ the global dimension of $R$ is equal to the supremum of $\operatorname{pd}(R / \mathfrak{m})$, where $\mathfrak{m}$ ranges over the maximal ideals of $R$ (see [24, (5.92)]). It has also been shown by K. R. Goodearl [12, Theorem 16] and S. M. Bhatwadekar [3, Proposition 1.1] that for a (left and right) noetherian ring $R$ whose global dimension is finite, the global dimension of $R$ is the supremum of $\operatorname{pd}(R / \mathfrak{m})$ where $\mathfrak{m}$ ranges over the maximal right ideals of $R$. It is an open question whether the finiteness of the global dimension can be dropped [13, Appendix]. 


\section{A Noncommutative Generalization of Kaplansky's Theorem}

The goal of this section is to prove a noncommutative generalization of Kaplansky's Theorem 1.2. Specifically, we shall show in Theorem 7.9 that a noetherian ring whose maximal right ideals are all principal is a principal right ideal ring. To motivate our approach, we shall recall a result [10, Theorem C] of A. W. Goldie: a left noetherian principal right ideal ring is a direct sum of a semiprime ring and an artinian ring. Inspired by this fact, our proof of Theorem 7.9 will proceed by taking noetherian ring whose maximal right ideals are principal and decomposing it as a direct sum of a semiprime ring and an artinian ring. This should seem reasonable because we have already shown in Corollary 5.5 that, in order to test whether an artinian ring is a PRIR, it suffices to test only its maximal right ideals.

With Goldie's result in mind, we begin this section by investigating under what conditions one can check the PRIR condition on a semiprime ring by testing only its maximal right ideals. The first result applies to semiprime rings with small right Krull dimension.

Proposition 7.1 Let $R$ be a semiprime ring with $\mathrm{r} . \mathrm{K} . \operatorname{dim}(R) \leq 1$. Then $R$ is a principal right ideal ring iff its maximal right ideals are principal.

Proof ("If" direction) By Theorem 6.11, it suffices to show that the essential cocritical right ideals of $R$ are principal. Thus it is enough to show that every essential cocritical right ideal of $R$ is maximal. According to [30,6.3.10] the fact that $R$ is semiprime with right Krull dimension means that, for every $E_{R} \subseteq R, \mathrm{~K} \cdot \operatorname{dim}(R / E)<$ $\mathrm{K} . \operatorname{dim}\left(R_{R}\right)=1$. So K. $\operatorname{dim}(R / E) \leq 0$, and if $E$ is also cocritical then it is 0 -cocritical and thus is maximal. This completes the proof.

In Example 7.11 below we will show that the hypothesis on the right Krull dimension cannot be relaxed. Of course, it is not the case that every semiprime PRIR has right Krull dimension $\leq 1$. In fact, in [14, Example 10.3] it is shown (using a construction of A. V. Jategaonkar from [17]) that there exist principal right ideal domains whose right Krull dimension is equal to any prescribed ordinal! So while Proposition 7.1 gives a sufficient condition for semiprime rings to be PRIRs, it is certainly not a necessary condition. However, with some additional effort we will use this result to formulate a precise characterization of semiprime left and right principal ideal rings in Corollary 7.5 below.

We will show in Proposition 7.4 below that if a semiprime ring with a certain finiteness condition on the left has all maximal right ideals principal, it must have small right Krull dimension. We take this opportunity to recall that a multiplicatively closed subset $S \subseteq R$ is saturated if, for any $a, b \in R, a b \in S$ implies $a, b \in S$.

Lemma 7.2 Let $R$ be a ring in which the multiplicative set of (resp. left) regular elements is saturated and which satisfies the ascending chain condition on left ideals of the form $R s$ where $s \in R$ is a (resp. left) regular element. Furthermore, suppose that every maximal right ideal of $R$ is principal. If $b \in R$ is a (resp. left) regular element, then $R / b R$ has finite length. 
Proof This argument adapts some of the basic ideas of factorization in noncommutative domains, as in Proposition 0.9.3 and Theorem 1.3.5 of [7]. However, we do not assume any of those results here.

If our fixed $b \in R$ is not right invertible, then $b R \neq R$. If $b R$ is not maximal, choose a maximal right ideal $a_{1} R \subsetneq R$ such that $b R \subsetneq a_{1} R$. Then $b=a_{1} b_{1}$ for some $b_{1} \in R$. We claim that $R b \subseteq R b_{1}$ is strict. Indeed, assume for contradiction that $R b=R b_{1}$. Then we may write $b_{1}=u b$ for some $u \in R$. Thus $b=a_{1} b_{1}=a_{1} u b$, and because $b$ is (left) regular we have $a_{1} u=1$. This contradicts the fact that $a_{1} R$ is maximal. Hence $R b \subsetneq R b_{1}$.

Because the set of (left) regular elements is saturated, we may now replace $b$ above by $b_{1}$ and proceed inductively to write $b_{i-1}=a_{i} b_{i}$ (if $b_{i-1} R$ is not maximal) where $b_{i}$ is (left) regular and $a_{i} R$ is a maximal right ideal. By the ACC condition on $R$, the chain

$$
R b \subsetneq R b_{1} \subsetneq R b_{2} \subsetneq \cdots
$$

cannot continue indefinitely. So the process must terminate, say at $b_{n-1}=a_{n} b_{n}$. This means that $b_{n} R$ is a maximal right ideal. Writing $a_{n+1}:=b_{n}$, we have a factorization $b=a_{1} \cdots a_{n+1}$ where the right ideals $a_{i} R$ are maximal. Then in the filtration

$$
b R=\left(a_{1} \cdots a_{n+1}\right) R \subseteq\left(a_{1} \cdots a_{n}\right) R \subseteq \cdots \subseteq a_{1} R \subseteq R,
$$

each factor module $\left(a_{1} \cdots a_{j-1}\right) R /\left(a_{1} \cdots a_{j}\right) R$ is a homomorphic image of the simple module $R / a_{j} R$ (via left multiplication by $a_{1} \cdots a_{j-1}$ ) and thus is simple. This proves that $R / b R$ has finite length, as desired.

In light of the hypotheses assumed above, the following definition will be useful.

Definition 7.3 We will say that a ring $R$ satisfies left ACC-reg if it satisfies the ascending chain condition on left ideals of the form $R s$ where $s \in R$ is a regular element.

Proposition 7.4 Let $R$ be a semiprime ring with right Krull dimension that satisfies left ACC-reg. If all of the maximal right ideals of $R$ are principal, then $\mathrm{r} . \mathrm{K} \cdot \operatorname{dim}(R) \leq 1$ and $R$ is a principal right ideal ring.

Proof Because $R$ is semiprime and has right Krull dimension, it is right Goldie (see $[30,6.3 .5]$ ). This has two important consequences. First, the set of regular elements of $R$ is saturated (because it is the intersection of $R$ with the group of units in its semisimple right ring of quotients). Second, the essential right ideals of $R$ are precisely the right ideals containing a regular element (see [24, (11.13)]). Thus, for every $E_{R} \subseteq_{e} R, R / E$ has finite length by Lemma 7.2 and thus has Krull dimension at most 0 . Now $[30,6.3 .10]$ provides us with the following equation for $\mathrm{r}$. K. $\operatorname{dim}(R)$ (which is valid because $R$ is semiprime with right Krull dimension):

$$
\text { r. K. } \operatorname{dim}(R)=\sup \left\{\mathrm{K} \cdot \operatorname{dim}(R / E)+1: E_{R} \subseteq_{e} R\right\} \leq 1 .
$$

Applying Proposition 7.1, we see that $R$ is a principal right ideal ring.

An immediate consequence is the aforementioned characterization of semiprime PIRs. 
Corollary 7.5 Let $R$ be a semiprime ring.

(1) $R$ is a principal ideal ring iff its left and right Krull dimensions are both at most 1 and the maximal left ideals and maximal right ideals of $R$ are all principal.

(2) Suppose that $R$ satisfies left $A C C$-reg. Then $R$ is a principal right ideal ring iff $\mathrm{r} . \mathrm{K} \cdot \operatorname{dim}(R) \leq 1$ and the maximal right ideals of $R$ are principal.

It is possible to strengthen Proposition 7.4 to show that more general types of rings must have small right Krull dimension.

Corollary 7.6 Let $R$ be a ring with right Krull dimension, and let $N$ be its prime radical. Suppose that one of the following two conditions holds:

(A) $R / N$ satisfies left ACC-reg;

(B) $R / P$ satisfies left ACC-reg for every minimal prime ideal $P \triangleleft R$.

If the maximal right ideals of $R$ are principal, then $\mathrm{r} . \mathrm{K} \cdot \operatorname{dim}(R) \leq 1$. In particular, $a$ noetherian ring whose maximal right ideals are principal has right Krull dimension at most 1 .

Proof According to [30, 6.3.8], the ring $R$ with right Krull dimension has finitely many minimal prime ideals $P_{1}, \ldots, P_{n}$ and

$$
\text { r. K. } \operatorname{dim}(R)=\text { r. K. } \operatorname{dim}(R / N)=\max \left\{\text { r. K. } \operatorname{dim}\left(R / P_{i}\right)\right\} .
$$

Because every factor ring of $R$ again has principal maximal right ideals, we may now apply Proposition 7.4.

It is an open question whether the left and right Krull dimensions of a general noetherian ring must be equal [13, Appendix]. However, another application of Proposition 7.4 shows that the Krull dimension of a noetherian PRIR must is symmetric.

Corollary 7.7 A left noetherian principal right ideal ring $R$ has

1. K. $\operatorname{dim}(R)=$ r. K. $\operatorname{dim}(R) \leq 1$.

Proof As mentioned before, the Krull dimension of $R$ is not changed upon factoring out its nilradical [30, 6.3.8]; thus we may assume that $R$ is semiprime. In this case, a result of J.C. Robson [34, Corollary 3.7] states that because $R$ is a noetherian PRIR, it must also be a PLIR. According to Proposition 7.4, both 1 . K. $\operatorname{dim}(R)$ and r. K. $\operatorname{dim}(R)$ are at most 1 . Now $R$ has Krull dimension 0 on either side precisely when $R$ is artinian on that side. But a noetherian ring is artinian on one side iff it is artinian on the other side. (This follows, for instance, from the Hopkins-Levitzki Theorem $[25,(4.15)]$.) Thus we see that the left and right Krull dimensions of $R$ must coincide, both equal to 0 when $R$ is artinian and both equal to 1 when $R$ is not artinian.

The next preparatory result provides a method of testing whether a module over a semilocal ring is zero. One may think of this as a variation of Nakayama's Lemma (even though the latter is used in the proof below). 
Lemma 7.8 Let $R$ be a semilocal ring, and let ${ }_{R} B$ be a finitely generated left module. If $B=\mathfrak{m} B$ for all maximal right ideals $\mathfrak{m}$ of $R$, then $B=0$.

Proof Let $R$ and ${ }_{R} B$ be as above, and let $J=\operatorname{rad}(R)$. We claim that $B / J B$ satisfies the same hypotheses over the semisimple ring $R / J$. Indeed, the maximal right ideals of $R / J$ are the right ideals of the form $\mathfrak{m} / J$ for a maximal right ideal $\mathfrak{m}$ of $R$. For such $\mathfrak{m} / J$ we have

$$
(\mathfrak{m} / J) \cdot(B / J B)=\mathfrak{m} B / J B=B / J B .
$$

Also, $B / J B$ is finitely generated over $R / J$. So $B / J B$ indeed satisfies the same hypotheses over $R / J$. If we knew the lemma to hold over all semisimple rings, it would follow that $B / J B=0$. Nakayama's Lemma would then imply that $B=0$.

So we may assume that $R$ is semisimple. Choose orthogonal idempotents $e_{1}, \ldots, e_{n}$ in $R$ whose sum is 1 such that $R_{R}=\bigoplus e_{i} R$ is a decomposition of $R$ into minimal right ideals. Then for any $k,\left(1-e_{k}\right) R=\bigoplus_{i \neq k} e_{i} R$ is a maximal right ideal of $R$. By hypothesis, we have $B=\left(1-e_{k}\right) R B=\left(1-e_{k}\right) B$. Because the $e_{i}$ are orthogonal,

$$
\left(1-e_{1}\right) \cdots\left(1-e_{k}\right)=1-\left(e_{1}+\cdots+e_{k}\right)
$$

In particular, $\left(1-e_{1}\right) \cdots\left(1-e_{n}\right)=1-\left(e_{1}+\cdots+e_{n}\right)=0$. It follows that

$$
B=\left(1-e_{1}\right) B=\left(1-e_{1}\right)\left(1-e_{2}\right) B=\cdots=\left(1-e_{1}\right) \cdots\left(1-e_{n}\right) B=0 .
$$

Let us review some relevant results on noetherian rings. For an ideal $I$ of a ring $R$, we let $\mathcal{C}(I)$ denote the set of elements $c \in R$ such that $c+I$ is a regular element of $R / I$. A theorem of J. C. Robson [35] states that a noetherian ring $R$ with prime radical $N$ is a direct sum of a semiprime ring and an artinian ring iff, for every $c \in$ $\mathcal{C}(N), N=c N=N c$. However, Robson commented in [35, p. 346] that if one only assumes that $N=c N$ for all $c \in \mathcal{C}(N)$, one can still conclude that there exists an idempotent $e \in R$ such that $e$ Re is semiprime, $(1-e) R(1-e)$ is artinian, and $e R(1-$ $e)=0$. This gives a useful "triangular decomposition" of such a ring. In particular it can be used to derive the result of Goldie, mentioned at the beginning of this section, that a left noetherian principal right ideal ring is a direct sum of a semiprime ring and an artinian ring. The first paragraph of our argument below borrows from the proof of this last statement given in [35, Theorem 4].

With all of the above results and remarks in hand, we are finally ready to prove our noncommutative generalization of Kaplansky's Theorem 1.2.

Theorem 7.9 (A noncommutative Kaplansky's Theorem) A noetherian ring is a principal right ideal ring iff its maximal right ideals are principal.

Proof ("If" direction) Suppose $R$ is a noetherian ring whose maximal right ideals are principal. Notice that every factor ring of $R$ satisfies the same hypotheses. Let $N \triangleleft R$ be the prime radical of $R$. We claim that $N=c N$ for every $c \in \mathcal{C}(N)$. Let $x \mapsto \bar{x}$ denote the canonical map $R \rightarrow R / N=: \bar{R}$. By Proposition 7.4, r. K. $\operatorname{dim}(\bar{R}) \leq$ 1. For $c \in \mathcal{C}(N)$, the element $\bar{c} \in \bar{R}$ is regular. So by [30, 6.3.9] we must have $\mathrm{K} \cdot \operatorname{dim}(\bar{R} / \bar{c} \bar{R})<\mathrm{K} \cdot \operatorname{dim}(\bar{R}) \leq 1$. So the right $R$-module $R /(N+c R) \cong \bar{R} / \bar{c} \bar{R}$ has Krull dimension at most 0 and thus has finite length. Hence $R /(N+c R)$ has a finite 
filtration with factors isomorphic to $R / \mathfrak{m}_{i}$ for some maximal right ideals $\mathfrak{m}_{1}, \ldots, \mathfrak{m}_{p}$ of $R$. The set of maximal right ideals of $R$ is certainly closed under similarity (it is the set of right ideals whose factor module is simple), so by Lemma 5.10 all maximal right ideals lie in the right Oka family $\mathcal{F}_{\mathrm{pr}}^{\circ}$. It follows from Corollary 2.6 that we have $N+c R \in \mathcal{F}_{\mathrm{pr}}^{\circ}$. Choose $d \in R$ such that $N+c R=d R$. Now in $\bar{R}$, $\bar{c} \bar{R}=\bar{d} \bar{R}$ means that $\bar{c}=\bar{d} \bar{r}$ for some $r \in R$. Because the set of regular elements in the semiprime noetherian $\operatorname{ring} \bar{R}$ is saturated, the fact that $c \in \mathcal{C}(N)$ implies that $d \in$ $\mathcal{C}(N)$. Now $N \subseteq d R$ implies that $N=d\left(d^{-1} N\right)$, and $d \in \mathcal{C}(N)$ gives $d^{-1} N=N$. Thus $N=d\left(d^{-1} N\right)=d N=(c R+N) N=c N+N^{2}$, and we conclude from Nakayama's Lemma $[25,(4.22)]$ (or by induction and the fact that $N$ is nilpotent) that $N=c N$.

Now according to Robson's decomposition result [35, p. 346] the ring $R$ is (up to isomorphism) of the form

$$
R=\left(\begin{array}{cc}
A & B \\
0 & S
\end{array}\right)
$$

where $A$ is an artinian ring, $S$ is a semiprime ring, and ${ }_{A} B_{S}$ is a (left and right noetherian) bimodule. Given any maximal right ideal $\mathfrak{m}$ of $A$, we will show that $B=\mathfrak{m} B$. The following is a maximal right ideal of $R$, and is therefore principal:

$$
\left(\begin{array}{cc}
\mathfrak{m} & B \\
0 & S
\end{array}\right)=\left(\begin{array}{ll}
x & y \\
0 & z
\end{array}\right) \cdot R
$$

for some $x \in \mathfrak{m}, y \in B$, and $z \in S$. It is easy to see that $z S=S$. Because $S$ is noetherian, $z$ must be a unit. Now for any $\beta \in B$, there exists $\left(\begin{array}{ll}a & b \\ 0 & c\end{array}\right) \in R$ such that

$$
\left(\begin{array}{ll}
x & y \\
0 & z
\end{array}\right)\left(\begin{array}{ll}
a & b \\
0 & c
\end{array}\right)=\left(\begin{array}{ll}
0 & \beta \\
0 & 0
\end{array}\right) \in\left(\begin{array}{cc}
\mathfrak{m} & B \\
0 & S
\end{array}\right) .
$$

Since $z c=0$ and $z$ is a unit, we must have $c=0$. Thus $\beta=x b \in \mathfrak{m} B$. Because $\beta \in B$ was arbitrary, this proves that $B=\mathfrak{m} B$. Since this holds for every maximal right ideal $\mathfrak{m}$ of $A$, we conclude from Lemma 7.8 that $B=0$.

Hence $R=A \oplus S$ where $A$ is an artinian ring and $S$ is a semiprime ring. The maximal right ideals of both $S$ and $A$ must also be principal. The artinian $\operatorname{ring} A$ is a PRIR according to Corollary 5.5, and it follows from Proposition 7.4 that the semiprime ring $S$ is a PRIR. It follows that $R=A \oplus S$ is a PRIR.

It is interesting to notice that, in the commutative setting, Kaplansky's Theorem 1.2 is "stronger" than the Kaplansky-Cohen Theorem 1.3, in the sense that Kaplansky originally derived Theorem 1.3 as a consequence of Theorem 1.2. This is opposite from our present situation, where the noncommutative version of the Kaplansky Theorem 7.9 in fact follows from (the "essential version" of) the noncommutative Kaplansky-Cohen Theorem 6.11 (through a series of other intermediate results).

The following example shows that Kaplansky's Theorem does not generalize if we remove the left noetherian hypothesis.

Example 7.10 A local right noetherian ring $R$ with right Krull dimension 1 whose unique maximal right ideal is principal, but which is not a principal right ideal ring. This construction is based on an exercise given in [26, Example 19.12]. Let $k$ be a field 
such that there exists a field isomorphism $\theta: k(x) \rightarrow k$ (which certainly does not fix $k)$, such as $k=\mathbb{Q}\left(x_{1}, x_{2}, \ldots\right)$. Consider the discrete valuation ring $A=k[x]_{(x)}$. Given a finitely generated module $M_{A}$, we define a $\operatorname{ring} R:=A \oplus M$ with multiplication given by

$$
(a, m) \cdot\left(a^{\prime}, m^{\prime}\right):=\left(a a^{\prime}, m^{\prime} \theta(a)+m a^{\prime}\right) .
$$

Let $\mathfrak{m}=x A \oplus M$ and $N=0 \oplus M$, both of which are ideals of $R$. Notice that $N^{2}=0$ while $\bar{R}:=R / N \cong A$ is a domain. This means that $N$ is the prime radical of $R$. Thus $N$ is contained in the Jacobson $\operatorname{radical} \operatorname{rad}(R)$. Because $R / \operatorname{rad}(R) \cong \bar{R} / \operatorname{rad}(\bar{R})$ is a field, the ring $R$ is local with Jacobson radical equal to $\mathfrak{m}$. Using the fact that $\theta(x) \in k$ is a unit in $A$, it is easy to conclude that $\mathfrak{m}=(x, 0) \cdot R$ is a principal right ideal.

Next we show that $R$ is right noetherian. Because the ring $R / N \cong A$ is noetherian, it is noetherian as a right $R$-module. Also, because $N^{2}=0$, the right $R$-action on $N_{R}=(0 \oplus M)_{R}$ factors through $R / N \cong A$. Because $A$ is noetherian and $M_{A}$ is finitely generated, this means that $N_{R}$ is noetherian. So $R_{R}$ is an extension of the noetherian right modules $R / N$ and $N$, proving that $R$ is right noetherian. Because the prime radical of $R$ is $N, \mathrm{r} . \mathrm{K} \cdot \operatorname{dim}(R)=\mathrm{r} . \mathrm{K} \cdot \operatorname{dim}(R / N)=\mathrm{K} \cdot \operatorname{dim}(A)=1$ (see [30, 6.3.8]). Finally, because the $R$-action on $N_{R}=(0 \oplus M)_{R}$ factors through $R / N \cong A$, if $M_{A}$ is any noncyclic $A$-module then $N$ is not principal as a right ideal in $R$. In fact, because the minimal number $\mu\left(N_{R}\right)$ of generators of $N_{R}$ is equal to $\mu\left(M_{A}\right)<\infty$, this number can be made as large as one desires.

Notice that the example above is not semiprime, in accordance with Proposition 7.1. With some extra work, we can produce a similar example $R$ that is a domain. By Proposition 7.1 again, we expect such $R$ to have right Krull dimension $>1$. (We thank G. M. Bergman for helping to correct an earlier, incorrect version of this example.)

Example 7.11 A local right noetherian domain $R$ with right Krull dimension 2 whose unique maximal right ideal is principal, but which is not a principal right ideal ring. Let $k, \theta: k(x) \stackrel{\sim}{\rightarrow} k$, and $A=k[x]_{(x)}$ be as in Example 7.10. Let $B=A[[y ; \theta]] \supseteq A$, the ring of skew power series over $A$ subject to the relation $a y=y \theta(a)$. Consider the ideal $I=y^{2} B$, and define the subring $R:=A \oplus I \subseteq B$. (Notice that $R$ is the subring of $B$ consisting of power series in which $y$ does not appear with exponent 1 . We can suggestively write $R=A\left[\left[y^{2}, y^{3} ; \theta\right]\right]$, with the understanding that the equation $a y=y \theta(a)$ only has meaning via its consequences $a y^{n}=y \theta^{n}(a)$ for $n \geq 2$.) Being a subring of the domain $B, R$ itself is a domain.

We claim that $I \subseteq \operatorname{rad}(R)$. It suffices to show that $1+I \subseteq U(R)($ see $[25,(4.5)])$. Let $i \in I$; then $1+i$ is a unit of $B$ because $I \subseteq y B=\operatorname{rad}(B)$. For $i^{\prime}:=-(1+i)^{-1} i=$ $-i(1+i)^{-1} \in I$ (note: $(1+i)^{-1}$ commutes with $i$ because $1+i$ does), we have

$$
(1+i) \cdot\left(1+i^{\prime}\right)=1+i+(1+i) i^{\prime}=1,
$$

and similarly $\left(1+i^{\prime}\right)(1+i)=1$. So $1+i \in U(R)$ as desired. One can now proceed as in Example 7.10 to show that $R$ is a local ring whose unique maximal right ideal $\mathfrak{m}:=x A \oplus I=x R$ is principal.

It is easy to see that $R$ is a free right module over the subring $A\left[\left[y^{2} ; \theta\right]\right] \cong$ $A[[t ; \theta]]=: S$ with basis $\left\{1, y^{3}\right\}$. Because $S$ is right noetherian (in fact, a principal right ideal domain, according to [16]), $R$ is also right noetherian. We claim that 
r. K. $\operatorname{dim}(S)=2$. First we show that for every $f \in S \backslash\{0\}, \mathrm{K} . \operatorname{dim}(S / f S) \leq 1$. Indeed, we can write $f=t^{m} x^{n} u$ for some unit $u \in S$. It follows from the filtration

$$
S \supseteq t S \supseteq t^{2} S \supseteq \cdots \supseteq t^{m} S \supseteq t^{m} x S \supseteq t^{m} x^{2} S \supseteq \cdots \supseteq t^{m} x^{n} S=f S
$$

that $S / f S$ has a filtration whose factors are isomorhpic to either $S / t S \cong A$ or $S / x S \cong$ $A / x A$. These filtration factors have submodule lattices isomorphic to that of $A_{A}$ or $(A / x A)_{A}$, and thus respectively have Krull dimension 1 or 0 . Hence K. $\operatorname{dim}(S / f S) \leq$ 1 as claimed. Because $S$ has right Krull dimension and is a domain, we see from Proposition 3.13 that $S_{S}$ is a critical module. We conclude that r. K. $\operatorname{dim}(S)=2$. Thus $\mathrm{K} \cdot \operatorname{dim}\left(R_{S}\right)=\mathrm{K} \cdot \operatorname{dim}\left(S_{S}^{2}\right)=\mathrm{K} \cdot \operatorname{dim}\left(S_{S}\right)=2$ (the second equality follows from the exact sequence $\left.0 \rightarrow S \rightarrow S^{2} \rightarrow S \rightarrow 0\right)$, which implies that $\mathrm{K} \cdot \operatorname{dim}\left(R_{R}\right) \leq \mathrm{K} \cdot \operatorname{dim}\left(R_{S}\right)=$ 2. On the other hand, the descending chain $I \supseteq I^{2} \supseteq I^{3} \supseteq \cdots$ of right ideals in $R$ has filtration factors $I^{m} / I^{m+1}=y^{2 m} B / y^{2 m+2} B \cong A \oplus A$. These have Krull dimension 1 , so we find $\mathrm{K} \cdot \operatorname{dim}\left(R_{R}\right)>1$ and thus r. K. $\operatorname{dim}(R)=2$.

Finally, we show that $I$ is not a principal right ideal of $R$. It suffices to show that $I / I \mathfrak{m}$ is not a cyclic right module over $R / \mathfrak{m} \cong k$. Notice that $I \mathfrak{m}=I(A x+I)=$ $I x+I^{2}$. Now $B y \subseteq y B$ implies that $I^{2}=\left(y^{2} B\right)^{2}=y^{4} B$. Also, $I x=y^{2} x A \oplus y^{3} x A \oplus$ $y^{4} x A \cdots$ Thus $I \mathfrak{m}=I x+I^{2}=y^{2} x A \oplus y^{3} x A \oplus y^{4} B$. It follows that

$$
\frac{I}{I \mathfrak{m}} \cong \frac{y^{2} A[[y ; \theta]]}{y^{2} x A \oplus y^{3} x A \oplus y^{4} A[[y ; \theta]]} \cong y^{2} k \oplus y^{3} k
$$

is not a cyclic $k$-vector space, as desired.

We conclude this section with some questions that arise in light of the results above. Examples 7.10 and 7.11 show that the left noetherian hypothesis in Theorem 7.9 cannot simply be dropped. While it seems somehow unnatural to try to omit the right noetherian hypothesis, we have not found an example showing this to be impossible. Thus we ask the following.

Question 7.12 Does there exist a left (but not right) noetherian ring $R$ whose maximal right ideals are all principal, but which is not a principal right ideal ring? What if we assume, in addition, that $R$ has right Krull dimension?

While reading an earlier draft of this work, G. M. Bergman kindly pointed out to us that no such example exists if we assume further that $R$ is a domain. We were able to generalize this to include semiprime right Goldie rings as follows.

Proposition 7.13 Let $R$ be a semiprime left noetherian ring in which every essential right ideal contains a regular element (the latter hypothesis is satisfied if $R$ is a domain or if $R$ is right Goldie-in particular, if $R$ has right Krull dimension). If every maximal right ideal of $R$ is principal, then $R$ is a principal right ideal ring.

Proof By Example 6.4, it is enough to show that every essential right ideal of $R$ is principal. To this end, fix $E_{R} \subseteq_{e} R$. Because $R$ has a semisimple left ring of quotients, the multiplicative set of regular elements of $R$ is saturated. Thus the hypotheses of Lemma 7.2 are satisfied. Since $E$ contains a regular element, that lemma implies that $R / E$ has finite length. So $R / E$ has a finite filtration whose factors are isomorphic to $R / \mathfrak{m}_{i}$ for some maximal right ideals $\mathfrak{m}_{1}, \ldots, \mathfrak{m}_{n}$ of $R$. Since the set of maximal right 
ideals is closed under similarity, Lemma 5.10 implies that all maximal right ideals of $R$ lie in $\mathcal{F}_{\mathrm{pr}}^{\circ}$. Now Corollary 2.6 implies that $E \in \mathcal{F}_{\mathrm{pr}}^{\circ} \subseteq \mathcal{F}_{\mathrm{pr}}$, so that $E$ is principal.

We also ask to what extent the PRIR condition can be tested up to similarity.

Question 7.14 Suppose that $R$ is a noetherian ring each of whose maximal right ideals is similar to a principal right ideal. Is $R$ a principal right ideal ring? If not, then is every right ideal of $R$ similar to a principal right ideal?

It would be interesting to test the status of the first Weyl algebra $R:=A_{1}(k)$ with respect to this question. Is every maximal right ideal of $R$ similar to a principal right ideal? Does $R$ have any right ideals that are not similar to principal right ideals? More generally, we wonder whether there exists any ring that is not a PRIR, but in which every right ideal is similar to a principal right ideal.

\section{Previous Generalizations of the Cohen and Kaplansky Theorems}

In this final section we will discuss how Theorems 4.5 and 5.11 relate to earlier noncommutative generalizations of the Cohen and Kaplansky-Cohen theorems in the literature. (We are not aware of any previous generalizations of Kaplansky's Theorem 1.2.) In [21], K. Koh generalized both of these theorems. He defined a right ideal $I_{R} \subsetneq R$ to be a "prime right ideal" if, for any right ideals $A, B \subseteq R$ such that $A I \subseteq I, A B \subseteq I$ implies that $A \subseteq I$ or $B \subseteq I$. Notice that this is equivalent to the condition that for $a, b \in R, a R b \subseteq I$ with $a R I \subseteq I$ imply that either $a \in I$ or $b \in I$. We will refer to such a right ideal as a Koh-prime right ideal. Koh showed that a ring $R$ is right noetherian (resp. a PRIR) iff all of its Koh-prime right ideals are finitely generated (resp. principal). Independently, in [4] (also appeared [5]) V.R. Chandran also gave generalizations of the Cohen and Kaplansky theorems, showing that a right duo ring is right noetherian (resp. a PRIR) iff all prime ideals of $R$ are finitely generated (whether this is f.g. as an ideal or f.g. as a right ideal is irrelevant, since $R$ is right duo). But Koh's result implies Chandran's result, since a two-sided ideal is Koh-prime as a right ideal iff it is a prime ideal in the usual sense.

Notice that our completely prime right ideals are necessarily Koh-prime right ideals. For suppose that $P_{R} \subseteq R$ is completely prime and that $A, B \subseteq R$ are such that $A P \subseteq P$ and $A B \subseteq P$. If $A \nsubseteq P$, then there exists $a \in A \backslash P$. Now $a P \subseteq P$, and for any $b \in B$ we have $a b \in P$. It follows that $b \in P$ because $P$ is completely prime. So $B \subseteq P$, proving that $P$ is Koh-prime. It follows that Theorems 4.5 and 5.11, with the set $\mathcal{S}$ taken to be the set of completely prime right ideals, imply Koh's theorems, which in turn imply Chandran's theorems.

On the other hand, G. O. Michler offered another noncommutative generalization of Cohen's Theorem in [31]. He defined a right ideal $I \subsetneq R$ to be "prime" if $a R b \subseteq I$ implies that either $a \in I$ or $b \in I$. This is equivalent to saying that, for right ideals $A, B \subseteq R, A B \subseteq I$ implies that one of $A$ or $B$ lies in $I$. We will refer to such right ideals as Michler-prime right ideals. Michler proved in [31] that a ring is right noetherian iff its Michler-prime right ideals are all finitely generated. Notice immediately that the Michler-prime right ideals of a given ring form a subset of 
the set of all Koh-prime right ideals of that ring; thus Michler's version of Cohen's Theorem generalizes Koh's version.

If we were to try to recover Michler's theorem directly from Theorem 4.5, we would need to check that the Michler-prime right ideals form a noetherian point annihilator set over an arbitrary ring $R$. In order to settle whether or not this is true, we offer an alternate description of the Michler-prime right ideals below. Recall that a module $M_{R} \neq 0$ is said to be a prime module if, for every nonzero submodule $N \subseteq$ $M$, $\operatorname{ann}(N)=\operatorname{ann}(M)$. One can show that the annihilator of a prime module is a prime ideal (for example, as in [24, (3.54)]).

Proposition 8.1 A right ideal $P \subsetneq R$ is Michler-prime iff $R / P$ is a prime module.

Proof First suppose that $P$ is Michler-prime. To see that $R / P$ is a prime module, consider a nonzero submodule $A / P \subseteq R / P$ (so that the right ideal $A$ properly contains $P)$. Denote $B:=\operatorname{ann}(A / P) \triangleleft R$. Then $(A / P) \cdot B=0$ implies that $A B \subseteq P$. Because $P$ is Michler-prime, this means that $B \subseteq P$, so that $(R / P) \cdot B=(P+B) / P=0$. So $B=\operatorname{ann}(R / P)$, proving that the module $R / P$ is prime.

Conversely, suppose that $R / P$ is a prime module. Let $a, b \in R$ be such that $a R b \subseteq P$ and $a \notin P$. It follows that $b$ annihilates $(P+a R) / P \neq 0$, so that $b \in$ $\operatorname{ann}((P+a R) / P)=\operatorname{ann}(R / P)$. In particular, $(R / P) \cdot b=0$ implies that $b \in P$. This proves that $P$ is Michler-prime.

Corollary 8.2 For a ring $R$, the set $\mathcal{S}$ of Michler-prime right ideals is a noetherian point annihilator set iff every nonzero noetherian right $R$-module has a prime submodule. This is satisfied, in particular, if $R$ has the ACC on ideals.

Proof The "only if" direction is clear from Proposition 8.1. For the "if" direction, let $M_{R}$ be any module with a prime submodule $N$. Notice that a nonzero submodule of a prime module is prime. Thus for any nonzero element $m \in N, R / \operatorname{ann}(m) \cong m R \subseteq N$ is a prime module. By Proposition 8.1, $\operatorname{ann}(m)$ is a Michler-prime right ideal. So if every nonzero noetherian module has a prime submodule, the set $\mathcal{S}$ is a noetherian point annihilator set.

If $R$ satisfies ACC on ideals, then every nonzero right $R$-module has a prime submodule-see [24, (3.58)]. So in this case $\mathcal{S}$ is a point annihilator set, hence a noetherian point annihilator set.

We conclude from Corollary 8.2 and Theorem 4.5 that a ring is right noetherian iff it satisfies ACC on ideals and all of its Michler-prime right ideals are finitely generated. This is actually a slight generalization of [31, Lemma 3], which Michler used as a "stepping stone" to prove his main result.

Nevertheless, there do exist nonzero noetherian modules over some (large) rings which do not have any prime submodules. Thus Michler's primes do not form a noetherian point annihilator set in every ring. We include an example below.

Example 8.3 Let $k$ be a division ring, and let $R$ be the ring of $\mathbb{N} \times \mathbb{N}$ row-finite upper triangular matrices over $k$. Let $M_{R}=\bigoplus_{\mathbb{N}} k$, viewed as row vectors over $k$, with the 
obvious right $R$-action. Let $M_{i}$ denote the submodule of $M$ consisting of row vectors whose first $i$ entries are zero. Then one can show that

$$
M=M_{0} \supsetneq M_{1} \supsetneq M_{2} \supsetneq \cdots
$$

are the only nonzero submodules of $M$. This visibly shows that $M$ is noetherian. (Indeed, one can say more: every submodule of $M$ is actually principal, generated by one of the "standard basis vectors." We omit the details because we will not use this fact.) However, one can see that ann $\left(M_{i}\right)$ is equal to the set of all matrices in $R$ whose first $i$ rows are arbitrary and whose other rows are zero. So the fact that

$$
\operatorname{ann}\left(M_{0}\right) \subsetneq \operatorname{ann}\left(M_{1}\right) \subsetneq \operatorname{ann}\left(M_{2}\right) \subsetneq \cdots
$$

makes it clear that $M$ has no prime submodules.

Incidentally, $M_{R}$ is also an example of a cyclic 1-critical module that is not a prime module. Thus, choosing a right ideal $I_{R} \subseteq R$ such that $R / I \cong M$ (such as the right ideal of matrices in $R$ whose first row is zero), we see that $I$ is cocritical but not Michler-prime.

In spite of this complication, it is in fact possible to derive Michler's Theorem from Theorem 4.5. The key observation that makes this possible is a lemma [37, Lemma 2] due to P.F. Smith. This result states that if every ideal of a ring $R$ contains a finite product of prime ideals each containing that ideal, and if $R$ satisfies the ACC on prime ideals, then every nonzero right $R$-module has a prime submodule.

Theorem 8.4 (Michler) $A$ ring $R$ is right noetherian iff all of the Michler-prime right ideals of $R$ are finitely generated.

Proof ("If" direction.) Suppose that the Michler-prime right ideals of $R$ are all finitely generated. Every prime (two-sided) ideal of $R$ is Michler-prime, and thus is finitely generated as a right ideal. By [31, Lemmas 4 \& 5] the following two conditions hold:

1. Every ideal $I \triangleleft R$ contains a product of finitely many prime ideals of $R$, where each of these ideals contains $I$;

2. $R$ satisfies the ascending chain condition on prime ideals.

It follows from [37, Lemma 2] that every nonzero right $R$-module has a prime submodule. So by Corollary 8.2, the set of Michler-prime right ideals is a noetherian point annihilator set for $R$. Now it follows from Theorem 4.5 that $R$ is a right noetherian ring.

In addition, our methods allow us to produce a generalization of the KaplanskyCohen Theorem that is in the spirit of Michler's Theorem! Note that this was not proved in [31], and in fact seems to be a new result.

Theorem 8.5 $A$ ring $R$ is a principal right ideal ring iff all of the Michler-prime right ideals of $R$ are principal.

Proof ("If" direction.) Suppose that all of the Michler-prime right ideals of $R$ are principal. As in the proof of Theorem 8.4 above, it follows that the set $\mathcal{S}$ of 
Michler-prime right ideals of $R$ is a noetherian point annihilator set for $R$. This set is closed under similarity thanks to Proposition 8.1, so Theorem 5.11 implies that $R$ is a principal right ideal ring.

For a given ring $R$, the effectiveness of Michler's Theorem versus Theorem 4.5 with $\mathcal{S}$ taken to be the set of completely prime right ideals of $R$ depends on the scarcity or abundance of right ideals in $R$ from the "test set" in either theorem. For example, over a simple ring $R$, every nonzero right $R$-module is certainly prime. So every proper right ideal of $R$ will be Michler-prime. (In fact, Koh [20, Theorem 4.2] has shown even more: a ring $R$ is simple iff all of its proper right ideals are Michlerprime.) Thus for a simple ring $R$, Michler's theorem provides no advantage, as we would still need to test every right ideal to see whether $R$ is right noetherian. On the other hand, all right ideals of a ring $R$ are completely prime only if $R$ is a division ring (see [33, Proposition 2.11]). So outside of this trivial class of rings, we are guaranteed that Theorem 4.5 with $\mathcal{S}=$ \{completely prime right ideals $\}$ reduces the set of right ideals which we need to test in order to determine whether a ring is right noetherian. We can expect Theorem 4.5 to be increasingly effective when we take $\mathcal{S}$ to be either of the two smaller test sets in Eq. 3.1.

There is another variant of Cohen's Theorem for right fully bounded rings. (Recall that $R$ is right fully bounded if, for every prime ideal $P \triangleleft R$, every essential right ideal of $R / P$ contains a nonzero ideal of $R / P$ ). This result says that a right fully bounded ring is right noetherian iff all of its prime ideals are finitely generated as right ideals. A statement of this theorem is given in [23, p. 95], and it is attributed to G. O. Michler and L. W. Small independently. P. F. Smith provided a proof using homological methods in [38, Corollary 5] and an elementary proof in [39, Theorem 1]. (On a related note, [39] also features a version of Cohen's Theorem for modules over commutative rings.) If one is satisfied to deal with the subclass of PI rings, then this result can be proved via the approach of the present paper.

Theorem 8.6 (Michler-Small) A PI ring $R$ is right noetherian iff all of its prime ideals are finitely generated as right ideals.

Proof ("If" direction.) Let $R$ be a PI ring in which every prime ideal is finitely generated as a right ideal. By Theorem 4.5, to prove that $R$ is right noetherian it is enough to show that every nonzero noetherian right $R$-module $M_{R}$ has a nonzero cyclic finitely presented submodule. (This basically produces a noetherian point annihilator set of right ideals that are finitely generated.)

As in the proof of Theorem 8.4, every nonzero noetherian right $R$-module has a prime submodule, so it suffices to assume that $M$ is prime. In this case, $P:=$ $\operatorname{Ann}(M)$ is prime. By a result of Amitsur and Small [1, Proposition 3], because the prime PI ring $R / P$ has a faithful noetherian module $M$, it is a noetherian ring. A result of Cauchon on right fully bounded noetherian rings (see, for instance, [13, Theorem 9.10]) now implies that there is an embedding $R / P \hookrightarrow M^{n}$ for some integer $n \geq 1$, and we will identify $R / P$ with its image as a submodule of $M$. Let $\pi: M^{n} \rightarrow$ $M$ be the projection of $M^{n}$ onto one of its components such that $R / P \nsubseteq \operatorname{ker} \pi$. The module $(R / P)_{R}$ is finitely presented (because $P_{R}$ is finitely generated) and noetherian. Thus $\pi(R / P) \subseteq M$ is a factor of the finitely presented module $R / P$ by a finitely generated submodule and must istelf be finitely presented (for instance, 
see $[24,(4.26)(b)])$. Hence $M$ has a nonzero cyclic finitely presented submodule as desired.

It is clear that the same proof would recover the Michler-Small Theorem for right fully bounded rings (not only PI rings) if the following question has an affirmative answer.

Question 8.7 Let $R$ is a prime right (fully) bounded ring with a faithful (prime) noetherian module $M_{R}$. Is $R$ right noetherian, or equivalently, is there an embedding $R_{R} \hookrightarrow M^{n}$ for some integer $n \geq 1$ ?

(The equivalence of the two questions follows from Cauchon's result, mentioned in the proof above, that a prime right fully bounded noetherian ring with a faithful module $M_{R}$ embeds into a finite direct sum of copies of $M$.)

In a more recent paper [40], B. V. Zabavs'kiǔ also studied noncommutative versions of the Cohen and Kaplansky-Cohen theorems. Theorem 1 of that paper states that, for a right chain ring $R$ (i.e., a ring whose right ideals are totally ordered under inclusion), if every Michler-prime right ideal is principal, then $R$ is a principal right ideal ring. This is clearly generalized by Theorem 8.5 above. There is second version of the Kaplansky-Cohen Theorem in [40, Theorem 2] using a test set that is equal to the set of Koh-prime right ideals. Thus this theorem is equivalent to Koh's theorem. A noncommutative Cohen's Theorem is proved in [40, Theorem 5] using a test set that contains the Michler-prime right ideals as a subset; thus this result is subsumed by Michler's Theorem. Finally, there are also some results in [40] investigating when every two-sided ideal of a ring is either finitely generated or principal when considered as a right ideal.

Acknowledgements I wish to thank T. Y. Lam for his guidance throughout the time that I worked on the topic at hand. He provided comments on a number of drafts of this work and helped to fix some of the terminology introduced here. I am grateful to George Bergman who read two drafts of this work and made many useful comments. In particular, he provided comments that helped to clarify the content of Section 4, as well as an effective suggestion to help repair an error in Example 7.11. I also thank W. Keith Nicholson for directing me to the reference [32] and for suggesting Definition 6.8 in order to clarify certain arguments in Section 6. Finally, I thank the referee for providing some helpful references.

Open Access This article is distributed under the terms of the Creative Commons Attribution Noncommercial License which permits any noncommercial use, distribution, and reproduction in any medium, provided the original author(s) and source are credited.

\section{References}

1. Amitsur, S.A., Small, L.W.: Finite-dimensional representations of PI algebras. J. Algebra 133(2), 244-248 (1990). MR 1067405 (91h:16029)

2. Beachy, J.A., Weakley, W.D.: A note on prime ideals which test injectivity. Commun. Algebra 15(3), 471-478 (1987). MR 882795 (88f:16026)

3. Bhatwadekar, S.M.: On the global dimension of some filtered algebras. J. Lond. Math. Soc. (2) 13(2), 239-248 (1976) MR 0404398 (53 \#8200)

4. Chandran, V.R.: On two analogues of Cohen's theorem. Indian J. Pure Appl. Math. 8(1), 54-59 (1977) MR 0453809 (56\#12062) 
5. Chandran, V.R.: On two analogues of Cohen's theorem. Pure Appl. Math. Sci. 7(1-2), 5-10 (1978) MR 0460378 (57 \#372)

6. Cohen, I.S.: Commutative rings with restricted minimum condition. Duke Math. J. 17, 27-42 (1950) MR $0033276(11,413 g)$

7. Cohn, P.M.: Free Ideal Rings and Localization in General Rings. New Mathematical Monographs, vol. 3. Cambridge University Press, Cambridge (2006) MR 2246388 (2007k:16020)

8. Eisenbud, D.: Commutative Algebra: With a View Toward Algebraic Geometry. Graduate Texts in Mathematics, vol. 150. Springer-Verlag, New York (1995) MR 1322960 (97a:13001)

9. Evans, E.G. Jr.: Krull-Schmidt and cancellation over local rings. Pac. J. Math. 46, 115-121 (1973) MR 0323815 (48 \#2170)

10. Goldie, A.W.: Non-commutative principal ideal rings. Arch. Math. 13, 213-221 (1962) MR 0140532 (25 \#3951)

11. Goldie, A.W.: Properties of the idealiser. In: Ring Theory (Proc. Conf., Park City, Utah, 1971), pp. 161-169. Academic Press, New York (1972) MR 0382341 (52 \#3226)

12. Goodearl, K.R.: Global dimension of differential operator rings. II. Trans. Am. Math. Soc. 209, 65-85 (1975) MR 0382359 (52 \#3244)

13. Goodearl, K.R., Warfield, R.B. Jr.: An Introduction to Noncommutative Noetherian Rings. London Mathematical Society Student Texts, vol. 61, 2nd edn. Cambridge University Press, Cambridge (2004) MR 2080008 (2005b:16001)

14. Gordon, R., Robson, J.C.: Krull Dimension. American Mathematical Society, no. 133. Memoirs of the American Mathematical Society, Providence (1973) MR 0352177 (50 \#4664)

15. Huynh, D.V.: A note on rings with chain conditions. Acta Math. Hungar. 51(1-2), 65-70 (1988) MR 934584 (89e:16024)

16. Jategaonkar, A.V.: Left principal ideal domains. J. Algebra 8, 148-155 (1968) MR 0218387 (36 $\# 1474)$

17. Jategaonkar, A.V.: A counter-example in ring theory and homological algebra. J. Algebra 12, 418-440 (1969) MR 0240131 (39 \#1485)

18. Kaplansky, I.: Elementary divisors and modules. Trans. Am. Math. Soc. 66, 464-491 (1949) MR $0031470(11,155 b)$

19. Kertész, A.: Noethersche ringe, die artinsch sind. Acta Sci. Math. (Szeged) 31, 219-221 (1970) MR 0279126 (43 \#4852)

20. Koh, K.: On one sided ideals of a prime type. Proc. Am. Math. Soc. 28, 321-329 (1971) MR 0274488 (43 \#251)

21. Koh, K.: On prime one-sided ideals. Can. Math. Bull. 14, 259-260 (1971) MR 0313325 (47 \#1880)

22. Koker, J.J.: Global dimension of rings with Krull dimension. Commun. Algebra 20(10), 28632876 (1992) MR 1179266 (94a:16011)

23. Krause, G.: On fully left bounded left noetherian rings. J. Algebra 23, 88-99 (1972) MR 0308188 (46 \#7303)

24. Lam, T.Y.: Lectures on Modules and Rings. Graduate Texts in Mathematics, vol. 189. SpringerVerlag, New York (1999) MR 1653294 (99i:16001)

25. Lam, T.Y.: A First Course in Noncommutative Rings. Graduate Texts in Mathematics, vol. 131, 2nd edn. Springer-Verlag, New York (2001) MR 1838439 (2002c:16001)

26. Lam, T.Y.: Exercises in Classical Ring Theory. Problem Books in Mathematics, 2nd edn. Springer-Verlag, New York (2003) MR 2003255 (2004g:16001)

27. Lam, T.Y.: A crash course on stable range, cancellation, substitution and exchange. J. Algebra Appl. 3(3), 301-343 (2004) MR 2096452 (2005g:16007)

28. Lam, T.Y.: Exercises in Modules and Rings. Problem Books in Mathematics. Springer, New York (2007) MR 2278849 (2007h:16001)

29. Lam, T.Y., Reyes, M.L.: A Prime Ideal Principle in commutative algebra. J. Algebra 319(7), 3006-3027 (2008) MR 2397420 (2009c:13003)

30. McConnell, J.C., Robson, J.C.: Noncommutative Noetherian Rings. Graduate Studies in Mathematics, vol. 30, revised ed. American Mathematical Society, Providence (2001) MR 1811901 (2001i:16039)

31. Michler, G.O.: Prime right ideals and right noetherian rings. In: Ring Theory (Proc. Conf., Park City, Utah, 1971), pp. 251-255. Academic Press, New York (1972) MR 0340334 (49 \#5089)

32. Osofsky, B.L.: A generalization of quasi-Frobenius rings. J. Algebra 4, 373-387 (1966) MR 0204463 (34 \#4305)

33. Reyes, M.L.: A one-sided Prime Ideal Principle for noncommutative rings. J. Algebra Appl. 9(6), 877-919 (2010) 
34. Robson, J.C.: Rings in which finitely generated right ideals are principal. Proc. Lond. Math. Soc. 17(3), 617-628 (1967) MR 0217109 (36 \#200)

35. Robson, J.C.: Decomposition of noetherian rings. Commun. Algebra 1, 345-349 (1974) MR 0342564 (49\#7310)

36. Rotman, J.J.: An Introduction to Homological Algebra. Pure and Applied Mathematics, vol. 85. Academic Press Inc. [Harcourt Brace Jovanovich Publishers], New York (1979) MR 538169 (80k:18001)

37. Smith, P.F.: Injective modules and prime ideals. Commun. Algebra 9(9), 989-999 (1981) MR 614468 (82h:16018)

38. Smith, P.F.: The injective test lemma in fully bounded rings. Commun. Algebra 9(17), 1701-1708 (1981) MR 631883 (82k:16030)

39. Smith, P.F.: Concerning a theorem of I. S. Cohen. An. Ştiinţ. Univ. Ovidius Constanţa Ser. Mat. 2, 160-167 (1994) XIth National Conference of Algebra (Constanţa, 1994). MR 1367558 (96m:16029)

40. Zabavs'kiř, B.V.: A noncommutative analogue of Cohen's theorem. Ukr. Mat. Z. 48(5), 707-710 (1996) MR 1417038 